\title{
A smash product construction of nonlocal vertex algebras
}

\author{
Haisheng $\mathrm{Li}^{1}$ \\ Department of Mathematical Sciences, Rutgers University, Camden, NJ 08102 \\ and \\ Department of Mathematics, Harbin Normal University, Harbin, China
}

\begin{abstract}
A notion of vertex bialgebra and a notion of module nonlocal vertex algebra for a vertex bialgebra are studied and then a smash product construction of nonlocal vertex algebras is presented. For every nonlocal vertex algebra $V$ satisfying a suitable condition, a canonical bialgebra $B(V)$ is constructed such that primitive elements of $B(V)$ are essentially pseudo derivations and group-like elements are essentially pseudo endomorphisms. Furthermore, vertex algebras associated with Heisenberg Lie algebras as well as those associated with nondegenerate even lattices are reconstructed through smash products.
\end{abstract}

\section{Introduction}

In the theory of Hopf algebras, there is a notion of $H$-module algebra for a bialgebra $H$, and furthermore for an $H$-module algebra $A$, one has the smash product algebra $A \sharp H$. The celebrated quantum groups (see $[\mathrm{Dr}],[\mathrm{J}]$ ) are (almost cocommutative) quasi-triangular Hopf algebras, which generalize Lie algebras and groups in the sense that the universal enveloping algebras of Lie algebras and the group algebras of groups are cocommutative Hopf algebras. In the theory of Hopf algebras, a very important role has been played by the Drinfeld's quantum double construction of quasi-triangular Hopf algebras. It was known (cf. [M]) that if $H$ is a finite-dimensional cocommutative Hopf algebra, the Drinfeld's quantum double $D(H)$ is isomorphic to the smash product $H^{*} \sharp H$ with respect to a specially defined action of $H$ on $H^{*}$. Presumably, suitable vertex analogues of the classical smash product construction and the Drinfeld quantum double construction will be of importance in the general theory of vertex algebras.

In this paper we formulate and study a notion of vertex bialgebra and a notion of $B$ module nonlocal vertex algebra for a vertex bialgebra $B$ and we then give a smash product construction. The vertex operator algebras associated with Heisenberg Lie algebras and the vertex algebras associated with nondegenerate even lattices are studied again in terms of the smash product construction. We shall study Drinfeld double construction in a sequel.

The notion of vertex bialgebra, studied in this paper, is rather simple, where a vertex bialgebra is just a nonlocal vertex algebra equipped with a (standard) coalgebra structure such that the comultiplication map and the counit map are homomorphisms of nonlocal vertex algebras. This notion is analogous to and generalizes the classical notion of

\footnotetext{
${ }^{1}$ Partially supported by an NSA grant
} 
bialgebra as the notion of nonlocal vertex algebra is analogous to and generalizes that of associative algebra. Two families of examples of vertex bialgebras are given in this paper. The first family is associated to standard bialgebras equipped with a derivation $\partial$ such that $\Delta \circ \partial=(\partial \otimes 1+1 \otimes \partial) \Delta$ and $\varepsilon \circ \partial=0$, and the second family of examples are based on vertex algebras associated with vertex Lie algebras ([DLM], [FB], [P]), namely conformal Lie algebras (see $[\mathrm{K}]$ ).

Note that a notion of vertex operator coalgebra, which is dual to the notion of vertex operator algebra, has been formulated and studied by Hubbard in [H1] and [H2]. We here just use the classical notion of coalgebra, bypassing the general notion of vertex operator coalgebra. Certainly, more general notions of vertex bialgebra exist.

For a vertex bialgebra $B$, a $B$-module nonlocal vertex algebra is a nonlocal vertex algebra $V$ equipped with a $B$-module structure such that the following conditions hold for $b \in B, v \in V$ :

$$
\begin{aligned}
& Y(b, x) v \in V \otimes \mathbb{C}((x)) \\
& Y(b, x) \mathbf{1}=\varepsilon(b) \mathbf{1} \\
& Y\left(b, x_{1}\right) Y(v, x)=\sum Y\left(Y\left(b_{(1)}, x_{1}-x\right) v, x\right) Y\left(b_{(2)}, x_{1}\right),
\end{aligned}
$$

where $\Delta(b)=\sum b_{(1)} \otimes b_{(2)}$. Furthermore, for a given vertex bialgebra $H$ and an $H$ module nonlocal vertex algebra $V$, the smash product nonlocal vertex algebra $V \sharp H$ has the underlying vector space $V \otimes H$ with the vertex operator map $Y^{\sharp}$ defined by

$$
Y^{\sharp}(u \otimes g, x)(v \otimes h)=\sum Y(u, x) Y\left(g_{(1)}, x\right) v \otimes Y\left(g_{(2)}, x\right) h
$$

for $u, v \in V, g, h \in H$. These notions (or construction) are analogous to and generalize their classical counterparts. For this study, a key role is played by the notions of pseudo derivation and pseudo endomorphism as we mention next.

In the work [EK on formal deformations of vertex operator algebras, Etingof and Kazhdan introduced and studied a notion of pseudo derivation, where a pseudo derivation of a vertex algebra $V$ is an element $\psi(x)$ of the space $\operatorname{Hom}_{\mathbb{C}}(V, V \otimes \mathbb{C}((x)))$ such that

$$
\begin{gathered}
{[L(-1), \psi(x)]=-\frac{d}{d x} \psi(x),} \\
\psi\left(x_{1}\right) Y\left(v, x_{2}\right)=Y\left(v, x_{2}\right) \psi\left(x_{1}\right)+Y\left(\psi\left(x_{1}+x_{2}\right) v, x_{2}\right) \quad \text { for } v \in V .
\end{gathered}
$$

A little bit earlier in [Li2], in an attempt to interpret the physics superselection theory in the context of vertex operator algebras, we studied objects $\Phi(x) \in \operatorname{Hom}\left(V, V\left[x, x^{-1}\right]\right)$ with the following properties:

$$
\begin{aligned}
& \Phi(x) \mathbf{1}=\mathbf{1}, \\
& {[L(-1), \Phi(x)]=-\frac{d}{d x} \Phi(x),} \\
& \Phi\left(x_{1}\right) Y(v, x)=Y\left(\Phi\left(x_{1}+x\right) v, x\right) \Phi\left(x_{1}\right) \quad \text { for all } v \in V .
\end{aligned}
$$


In view of the notion of pseudo derivation, such objects $\Phi(x)$ are nothing but "pseudo endomorphisms" and this observation was expanded in [Li5]. What was proved in Li2] (cf. [Li1]) that for such a $\Phi(x)$ and for any $V$-module $\left(W, Y_{W}\right)$, the pair $\left(W, Y_{W}(\Phi(x) \cdot, x)\right)$ carries the structure of a $V$-module. If $\Phi(x)$ is invertible, it was proved that $(V, Y(\Phi(x) \cdot, x))$ is a simple current in a certain sense. In that study, $\Phi(x)$ was indeed treated as a kind of endomorphism.

Recall that for a Hopf algebra $H$ with an $H$-module algebra $A$, primitive elements of $H$ act on $A$ by derivations while group-like elements of $H$ act on $A$ as automorphisms. For our particular notions of vertex bialgebra and module vertex algebra, the roles of derivations and automorphisms are played by pseudo derivations and pseudo automorphisms. What is more, for any nonlocal vertex algebra $V$ satisfying a mild condition, we construct a canonical bialgebra $B(V)$ whose primitive elements are essentially pseudo derivations and whose group-like elements are essentially pseudo automorphisms. By definition, $B(V)$ is the (unique) maximal subspace of $\operatorname{Hom}_{\mathbb{C}}(V, V \otimes \mathbb{C}((x)))$, satisfying a certain property. Note that $\operatorname{Hom}_{\mathbb{C}}(V, V \otimes \mathbb{C}((x)))$, which can be identified with $\operatorname{End}_{\mathbb{C}((x))}(V \otimes \mathbb{C}((x)))$, is an associative algebra over $\mathbb{C}((x))$. It is proved that for a general $V, B(V)$ is an associative subalgebra with the formal differential operator $d / d x$ as a $\mathbb{C}$-linear derivation. If $V$ is a simple vertex operator algebra in the sense of [FLM], or if $V$ is a vertex algebra with a certain basis of $\mathrm{P}-\mathrm{B}-\mathrm{W}$ type, it is proved that $B(V)$ is furthermore a bialgebra.

Just as a simple associative algebra cannot be made into a bialgebra, a simple vertex algebra cannot be made into a vertex bialgebra. On the other hand, as Borcherds pointed out in [B3], vertex algebras often have a natural cocommutative bialgebra structure, and furthermore, the canonical operator $L(-1)$ of the vertex algebras acts as a derivation. Then we have a natural vertex bialgebra. For example, for the vertex algebra $V_{L}$ associated with a nondegenerate even lattice $L$, the underlying vector space is the tensor product of a symmetric algebra with the group algebra of $L$, which is naturally a commutative and cocommutative Hopf algebra. This vertex bialgebra is denoted by $B_{L}$. Let $\epsilon$ be a 2-cocycle of $L$, which was used in the construction of the vertex algebra $V_{L}$ (see [FLM]) and let $B_{L, \epsilon}$ be the tensor product of the symmetric algebra with the $\epsilon$-twisted group algebra of $L$. Naturally, $B_{L, \epsilon}$ is a (non-commutative) associative algebra with $L(-1)$ as a derivation. Then $B_{L, \epsilon}$ becomes a nonlocal vertex algebra. It is proved that there is a natural $B_{L}$-module nonlocal vertex algebra structure on $B_{L, \epsilon}$ such that the smash product nonlocal vertex algebra $B_{L, \epsilon \sharp} B_{L}$ contains $V_{L}$ a subalgebra. We also obtain similar results for the vertex operator algebras associated with Heisenberg Lie algebras, where the vertex operator algebras are identified with the diagonal subalgebras of the smash products of type $B \sharp B$ with the comultiplication map as the vertex algebra isomorphism.

This paper is organized as follows: In Section 2, we study pseudo derivations and pseudo endomorphisms for nonlocal vertex algebras. In Section 3, we construct a canonical bialgebra for a nondegenerate nonlocal vertex algebra. In Section 4, we define the notion of vertex bialgebra and the notion of module nonlocal vertex algebra for a vertex bialgebra, and we give the smash product construction. In Section 5, we use the smash product construction to construct the vertex algebras associated with Heisenberg Lie algebras and the vertex algebras associated with nondegenerate even lattices. 


\section{Pseudo derivations and pseudo endomorphisms}

In this section we revisit pseudo-derivations and pseudo-endomorphisms for nonlocal vertex algebras, extending some of the results obtained in [Li5].

First, we recall the notion of nonlocal vertex algebra $([\mathrm{B} 2],[\mathrm{BK}],[\mathrm{Li} 3],[\mathrm{Li} 6])$. A nonlocal vertex algebra is a vector space $V$ equipped with a linear map

$$
\begin{aligned}
Y(\cdot, x): \quad & V \rightarrow \operatorname{Hom}(V, V((x))) \subset(\text { End } V)\left[\left[x, x^{-1}\right]\right] \\
& v \mapsto Y(v, x)=\sum_{n \in \mathbb{Z}} v_{n} x^{-n-1} \quad\left(\text { where } v_{n} \in \text { End } V\right)
\end{aligned}
$$

and equipped with a distinguished vector $\mathbf{1}$ of $V$, called the vacuum vector, such that

$$
\begin{aligned}
& Y(\mathbf{1}, x)=1, \\
& Y(v, x) \mathbf{1} \in V[[x]] \quad \text { and } \quad \lim _{x \rightarrow 0} Y(v, x) \mathbf{1}=v \quad \text { for } v \in V
\end{aligned}
$$

and such that for $u, v, w \in V$, there exists a nonnegative integer $l$ such that

$$
\left(x_{0}+x_{2}\right)^{l} Y\left(u, x_{0}+x_{2}\right) Y\left(v, x_{2}\right) w=\left(x_{0}+x_{2}\right)^{l} Y\left(Y\left(u, x_{0}\right) v, x_{2}\right) w .
$$

With this notion, an (ordinary) vertex algebra $V$ is simply a nonlocal vertex algebra that satisfies the weak commutativity in the sense that for any $u, v \in V$, there exists a nonnegative integer $k$ such that

$$
\left(x_{1}-x_{2}\right)^{k} Y\left(u, x_{1}\right) Y\left(v, x_{2}\right)=\left(x_{1}-x_{2}\right)^{k} Y\left(v, x_{2}\right) Y\left(u, x_{1}\right) .
$$

For a nonlocal vertex algebra $V$, just as with an ordinary vertex algebra, we define a linear operator $\mathcal{D}$ by $\mathcal{D} v=v_{-2} \mathbf{1}$ for $v \in V$. Then we have

$$
[\mathcal{D}, Y(v, x)]=Y(\mathcal{D} v, x)=\frac{d}{d x} Y(v, x) \quad \text { for } v \in V .
$$

If $V$ is a vertex operator algebra in the sense of [FLM] and [FHL, then $\mathcal{D}=L(-1)$.

Example 2.1. Let $A$ be any (unital) associative algebra equipped with a derivation $\partial$. Then (see $[\mathrm{B} 1,2])$ we have a nonlocal vertex algebra structure on $A$ with 1 as the vacuum vector and with

$$
Y(a, x) b=\left(e^{x \partial} a\right) b \quad \text { for } a, b \in A .
$$

In this case $\mathcal{D}=\partial$. We denote this nonlocal vertex algebra by $(A, \partial)$. Clearly, $(A, \partial)$ is an ordinary vertex algebra if and only if $A$ is commutative. On the other hand, let $V$ be a nonlocal vertex algebra such that $Y(u, x) v \in V[[x]]$ for all $u, v \in V$. Then one can prove that $V$ equipped with the multiplicative operation defined by $u \cdot v=u_{-1} v$ for $u, v \in V$ is an associative algebra with $\mathbf{1}$ as the identity and with $\mathcal{D}$ as a derivation. Furthermore, $Y(u, x) v=\left(e^{x \mathcal{D}} u\right) \cdot v$ for $u, v \in V$. 
Remark 2.2. Let $A$ and $B$ be (unital) associative algebras equipped with derivations $\partial_{A}$ and $\partial_{B}$, respectively. Then a linear map $f$ from $A$ to $B$ is a homomorphism of nonlocal vertex algebras from $\left(A, \partial_{A}\right)$ to $\left(B, \partial_{B}\right)$ if and only if $f$ is a homomorphism of algebras such that $f \partial_{A}=\partial_{B} f$.

Remark 2.3. Let $A$ and $B$ be (unital) associative algebras equipped with derivations $\partial_{A}$ and $\partial_{B}$, respectively. Clearly, $\partial_{A} \otimes 1+1 \otimes \partial_{B}$ is a derivation of the tensor product associative algebra $A \otimes B$. Then we have

$$
\left(A, \partial_{A}\right) \otimes\left(B, \partial_{B}\right)=\left(A \otimes B, \partial_{A} \otimes 1+1 \otimes \partial_{B}\right) .
$$

Let $V$ and $K$ be nonlocal vertex algebras. A homomorphism of nonlocal vertex algebras from $V$ to $K$ is a linear map $\psi$ from $V$ to $K$ such that

$$
\begin{aligned}
& \psi(\mathbf{1})=\mathbf{1}, \\
& \psi Y(u, x) v=Y(\psi(u), x) \psi(v) \quad \text { for } u, v \in V .
\end{aligned}
$$

It follows that $\psi \mathcal{D}_{V}=\mathcal{D}_{K} \psi$, where $\mathcal{D}_{V}$ and $\mathcal{D}_{K}$ denote the $\mathcal{D}$-operators of $V$ and $K$, respectively. A homomorphism of nonlocal vertex algebras from $V$ to itself is called an endomorphism of $V$.

Let $V$ be a nonlocal vertex algebra. A derivation of $V$ (see [B1]) is a linear endomorphism $f$ of $V$ such that

$$
f Y(v, x)=Y(f(v), x)+Y(v, x) f \quad \text { for } v \in V .
$$

All the derivations of $V$ form a Lie subalgebra $\operatorname{Der}(V)$ of the general linear Lie algebra $\mathfrak{g r}(V)$. If $V$ is an ordinary vertex algebra, for any $v \in V, v_{0}$ is a derivation of $V$ and $\left\{v_{0} \mid v \in V\right\}$ is an ideal of $\operatorname{Der}(V)$.

The following is a simple analogue of a classical notion:

Definition 2.4. Let $V$ and $K$ be nonlocal vertex algebras and let $\sigma$ and $\tau$ be homomorphisms of nonlocal vertex algebras from $V$ to $K$. A $(\sigma, \tau)$-derivation from $V$ to $K$ is a linear map $\psi$ from $V$ to $K$ such that for $u, v \in V$,

$$
\psi(Y(u, x) v)=Y(\sigma(u), x) \psi(v)+Y(\psi(u), x) \tau(v) .
$$

In the case that $V$ is a subalgebra of $K$ with $\sigma$ and $\tau$ being the identity homomorphism, we simply call a $(\sigma, \tau)$-derivation a derivation from $V$ to $K$. All the derivations from $V$ to $K$ form a subspace which we denote by $\operatorname{Der}(V, K)$.

For any derivation $f$ from $V$ to $K$, from (2.11) we have

$$
f(\mathbf{1})=0, \quad \mathcal{D}_{K} f=f \mathcal{D}_{V} .
$$

Now, let $K$ be the tensor product nonlocal vertex algebra $V \otimes(A, \partial)$, where $(A, \partial)$ is the nonlocal vertex algebra associated with an associative algebra $A$ equipped with a 
derivation $\partial$ (recall Example 2.1). Let $\sigma=\tau$ be the natural embedding of $V$ into $K$. We are interested in derivations from $V$ to $V \otimes(A, \partial)$. Consider $V \otimes A$ as an $A$-module in the obvious way. We have

$$
\operatorname{Hom}_{\mathbb{C}}(V, V \otimes A)=\operatorname{End}_{A}(V \otimes A)
$$

as $A$-modules and as $\mathbb{C}$-vector spaces. We may and we should consider any linear map from $V$ to $V \otimes A$ or from $V$ to $V$ as an $A$-linear endomorphism of $V \otimes A$. The vertex operator map $Y$ of $V$ is also considered as an $A$-linear map from $V \otimes A$ to $(\operatorname{End}(V \otimes A))\left[\left[x, x^{-1}\right]\right]$, that is,

$$
Y(v \otimes a, x)=Y(v, x) \otimes a \quad \text { for } v \in V, a \in A .
$$

On the other hand, we have the vertex operator map which we denote by $Y_{\text {ten }}$ for the tensor product nonlocal vertex algebra $V \otimes(A, \partial)$.

Lemma 2.5. Let $V$ be a nonlocal vertex algebra and let $A$ be an associative algebra equipped with a derivation $\partial$. A linear map $\psi$ from $V$ to $V \otimes A$ is a derivation from $V$ to the tensor product nonlocal vertex algebra $V \otimes(A, \partial)$ if and only if

$$
\psi Y(v, x)=Y(v, x) \psi+Y\left(e^{x(1 \otimes \partial)} \psi(v), x\right) \quad \text { for } v \in V .
$$

Proof. For $u \in V, a \in A$, we have

$$
Y_{\text {ten }}(u \otimes a, x)=Y(u, x) \otimes Y(a, x)=Y(u, x) \otimes\left(e^{x \partial} a\right)=Y\left(e^{x(1 \otimes \partial)}(u \otimes a), x\right) .
$$

Then

$$
Y_{\text {ten }}(\psi(v), x)=Y\left(e^{x(1 \otimes \partial)} \psi(v), x\right) \quad \text { for } v \in V .
$$

For $v \in V$, we see that $\psi Y(v, x)=Y(v, x) \psi+Y_{\text {ten }}(\psi(v), x)$ exactly amounts to

$$
\psi Y(v, x)=Y(v, x) \psi+Y\left(e^{x(1 \otimes \partial)} \psi(v), x\right) .
$$

This proves the assertion.

Now we consider special cases with $A=\mathbb{C}((x))$ or $\mathbb{C}((x))[\log x]$ and with $\partial= \pm d / d x$.

Definition 2.6. Let $V$ be a nonlocal vertex algebra. We define $\operatorname{PDer}^{ \pm}(V)$ to be the subspaces of $\operatorname{Hom}_{\mathbb{C}}(V, V \otimes \mathbb{C}((x)))$, consisting of elements $\psi(x)$ satisfying the condition

$$
\left[\psi\left(x_{1}\right), Y\left(v, x_{2}\right)\right]=Y\left(\psi\left(x_{1} \pm x_{2}\right) v, x_{2}\right) \quad \text { for all } v \in V \text {. }
$$

Proposition 2.7. Let $V$ be a nonlocal vertex algebra. We have

$$
\operatorname{PDer}^{ \pm}(V)=\operatorname{Der}(V, V \otimes(\mathbb{C}((x)), \pm d / d x)) .
$$

Furthermore, for $\psi(x) \in \operatorname{PDer}^{ \pm}(V)$, we have

$$
\psi(x) \mathbf{1}=0, \quad[\mathcal{D}, \psi(x)]=\mp \frac{d}{d x} \psi(x) .
$$


Proof. For $f(x) \in \mathbb{C}((x))$, we have $e^{ \pm x_{2} \frac{d}{d x}} f(x)=f\left(x \pm x_{2}\right)$ (by the Taylor theorem). Then for $v \in V$,

$$
Y\left(e^{ \pm x_{2}\left(1 \otimes \frac{d}{d x}\right)} \psi(x) v, x_{2}\right)=Y\left(\psi\left(x \pm x_{2}\right) v, x_{2}\right) .
$$

Now the first assertion follows from Lemma 2.5. For $\psi(x) \in \operatorname{PDer}^{ \pm}(V)$, since $\psi(x)$ is a derivation from $V$ to $V \otimes\left(\mathbb{C}((x)), \pm \frac{d}{d x}\right)$, we have $\psi(x) \mathbf{1}=0$ and

$$
\psi(x) \mathcal{D}_{V}=\left(\mathcal{D} \otimes 1 \pm 1 \otimes \frac{d}{d x}\right) \psi(x)
$$

noticing that the $\mathcal{D}$-operator of $V \otimes\left(\mathbb{C}((x)), \pm \frac{d}{d x}\right)$ is $\mathcal{D} \otimes 1 \pm 1 \otimes \frac{d}{d x}$. Then we have

$$
[\mathcal{D}, \psi(x)]=\mp \frac{d}{d x} \psi(x) \text { on } V
$$

completing the proof.

Remark 2.8. Recall from [EK] that a pseudo-derivation of an ordinary vertex algebra $V$ is a linear map $a(x)$ from $V$ to $V \otimes \mathbb{C}((x))$ such that

$$
\begin{aligned}
& {[\mathcal{D}, a(x)]=-\frac{d}{d x} a(x),} \\
& {\left[a\left(x_{1}\right), Y\left(v, x_{2}\right)\right]=Y\left(a\left(x_{1}+x_{2}\right) v, x_{2}\right) \quad \text { for all } v \in V .}
\end{aligned}
$$

The space of pseudo-derivations of $V$ was denoted by $\operatorname{PDer}(V)$. By Proposition 2.7. a pseudo-derivation of $V$ in the sense of Etingof and Kazhdan is exactly a derivation from $V$ to $V \otimes(\mathbb{C}((x)), d / d x)$.

Remark 2.9. Let $V$ be any nonlocal vertex algebra. Then $\operatorname{PDer}^{ \pm}(V)$ are subalgebras of the linear Lie algebra $\mathfrak{g l}(V \otimes \mathbb{C}((x)))$ and the formal differential operator $\frac{d}{d x}$ is a $\mathbb{C}$ linear derivation of $\operatorname{PDer}^{ \pm}(V)$. It is straightforward to see that the linear endomorphism of $\operatorname{Hom}(V, V \otimes \mathbb{C}((x)))$, sending $a(x)$ to $a(-x)$, is a Lie algebra isomorphism between $\operatorname{PDer}^{ \pm}(V)$.

We have the following result (cf. [EK]):

Proposition 2.10. Let $V$ be an ordinary vertex algebra. For any $u \in V, f(x) \in$ $\mathbb{C}((x))[\log x]$, set

$$
\Phi^{ \pm}(u, f)=\operatorname{Res}_{x_{1}} e^{ \pm x_{1} \frac{\partial}{\partial x}} f(x) Y\left(u, x_{1}\right)=\sum_{n \geq 0} \frac{( \pm 1)^{n}}{n !} f^{(n)}(x) u_{n} .
$$

Then

$$
\Phi^{ \pm}(u, f) \in \operatorname{Der}(V,(\mathbb{C}((x))[\log x], \pm d / d x)) .
$$

Furthermore, if $f(x) \in \mathbb{C}((x))$, then $\Phi^{ \pm}(u, f) \in \operatorname{PDer}^{ \pm}(V)$. In particular, for $u \in V$,

$$
Y(u, x)^{-}=\sum_{n \geq 0} u_{n} x^{-n-1}=\Phi^{-}\left(u, x^{-1}\right) \in \operatorname{PDer}^{-}(V) .
$$


Proof. For any $v \in V, f \in \mathbb{C}((x))[\log x]$, considering $v \otimes f$ as an element of the vertex algebra $V \otimes(\mathbb{C}((x))[\log x], \pm d / d x)$, we have

$$
(u \otimes f)_{0}=\operatorname{Res}_{x_{1}} Y_{t e n}\left(u \otimes f, x_{1}\right)=\operatorname{Res}_{x_{1}}\left(e^{ \pm x_{1} \frac{\partial}{\partial x}} f(x)\right) Y\left(u, x_{1}\right)=\Phi^{ \pm}(u, f) .
$$

Since $V \otimes(\mathbb{C}((x))[\log x], \pm d / d x)$ are ordinary vertex algebras, $(u \otimes f)_{0}$ is a derivation of $V \otimes(\mathbb{C}((x))[\log x], \pm d / d x)$ and hence a derivation from $V$ to $V \otimes(\mathbb{C}((x))[\log x], \pm d / d x)$. If $f(x) \in \mathbb{C}((x))$, by Proposition [2.7] $\Phi^{ \pm}(u, f) \in \operatorname{PDer}^{\mp}(V)$.

The elements $\Phi^{ \pm}(u, f)$ in Proposition 2.10 are somewhat inner pseudo derivations. If $V$ is a nonlocal vertex algebra associated to a differential associative algebra, such pseudo derivations are all zero. In this case, we have the following result:

Proposition 2.11. Let $A$ be an associative algebra equipped with a derivation $\partial$. Suppose that $d(x) \in(\operatorname{Der} A)\left[\left[x, x^{-1}\right]\right][\log x]$ such that

$$
\begin{aligned}
& d(x) a \in A \otimes \mathbb{C}((x))[\log x] \quad \text { for } a \in A, \\
& {[\partial, d(x)]= \pm \frac{d}{d x} d(x) .}
\end{aligned}
$$

Then $d(x) \in \operatorname{Der}\left(A, A \otimes\left(\mathbb{C}((x))[\log x], \mp \frac{d}{d x}\right)\right)$. Furthermore, if $d(x) \in(\operatorname{Der} A)\left[\left[x, x^{-1}\right]\right]$, we have $d(x) \in \operatorname{PDer}^{\mp}(A, \partial)$.

Proof. From the assumption we have

$$
e^{x_{1} \partial} d(x) e^{-x_{1} \partial}=e^{ \pm x_{1} \frac{d}{d x}} d(x)
$$

For $a, b \in A$, we have

$$
\begin{aligned}
d(x) Y\left(a, x_{2}\right) b & =d(x)\left(\left(e^{x_{2} \partial} a\right) b\right) \\
& =\left(d(x) e^{x_{2} \partial} a\right) b+\left(e^{x_{2} \partial} a\right) d(x) b \\
& =\left(e^{x_{2} \partial} e^{\mp x_{2} \frac{d}{d x}} d(x) a\right) b+\left(e^{x_{2} \partial} a\right) d(x) b \\
& =Y\left(e^{\mp x_{2} \frac{d}{d x}} d(x) a, x_{2}\right) b+Y\left(a, x_{2}\right) d(x) b .
\end{aligned}
$$

This proves $d(x) \in \operatorname{Der}\left(A, A \otimes\left(\mathbb{C}((x))[\log x], \mp \frac{d}{d x}\right)\right)$. The last assertion follows from Proposition 2.7

Next we study endomorphisms. We have the following straightforward analogue of Lemma 2.5.

Lemma 2.12. Let $V$ be a nonlocal vertex algebra and let $A$ be an associative algebra equipped with a derivation $\partial$. A linear map $\phi$ from $V$ to $V \otimes A$ is a homomorphism of nonlocal vertex algebras from $V$ to the tensor product nonlocal vertex algebra $V \otimes(A, \partial)$ if and only if

$$
\begin{aligned}
& \phi(\mathbf{1})=\mathbf{1} \\
& \phi Y(v, x)=Y\left(e^{x(1 \otimes \partial)} \phi(v), x_{2}\right) \phi \quad \text { for } v \in V .
\end{aligned}
$$


Definition 2.13. Let $V$ be a nonlocal vertex algebra. We define $\operatorname{PEnd}^{ \pm}(V)$ to be the subspaces of $\operatorname{Hom}_{\mathbb{C}}(V, V \otimes \mathbb{C}((x)))$, consisting of elements $\phi(x)$ satisfying the conditions

$$
\begin{aligned}
& \phi(x) \mathbf{1}=\mathbf{1} \\
& \phi\left(x_{1}\right) Y\left(v, x_{2}\right)=Y\left(\phi\left(x_{1} \pm x_{2}\right) v, x_{2}\right) \phi\left(x_{2}\right) \quad \text { for } v \in V .
\end{aligned}
$$

The following is an analogue of Proposition 2.7

Proposition 2.14. Let $V$ be a nonlocal vertex algebra and let $\phi(x) \in \operatorname{Hom}_{\mathbb{C}}(V, V \otimes \mathbb{C}((x)))$. Then $\phi(x) \in \mathrm{PEnd}^{ \pm}(V)$ if and only if $\phi(x)$ is a homomorphism of nonlocal vertex algebras from $V$ to $V \otimes(\mathbb{C}((x)), \pm d / d x)$. Furthermore,

$$
[\mathcal{D}, \phi(x)]=\mp \frac{d}{d x} \phi(x) \quad \text { for } \phi(x) \in \operatorname{PEnd}^{ \pm}(V) .
$$

The following is an explicit construction of pseudo endomorphisms (cf. [Li2]):

Proposition 2.15. Let $V$ be an ordinary vertex algebra and let $h \in V$ be such that

$$
[h(m), h(n)]=0 \quad \text { for } m, n \geq 0,
$$

where $h(k)=h_{k}$ for $k \in \mathbb{Z}$, and such that $h(0)$ acts semisimply on $V$ with integral eigenvalues. Then $E^{+}(-h, x) x^{h(0)} \in \mathrm{PEnd}^{-}(V)$, where

$$
E^{+}(-h, x)=\exp \left(\sum_{n \geq 1} \frac{-h(n)}{n} x^{-n}\right) \text {. }
$$

Proof. Set

$$
\phi(x)=h(0) \log x-\sum_{n \geq 1} \frac{h(n)}{n} x^{-n} \in \operatorname{Hom}(V, V \otimes \mathbb{C}((x))[\log x]) .
$$

Then

$$
\phi(x)=\sum_{n \geq 0} \frac{(-1)^{n}}{n !} h(n)\left(\frac{d}{d x}\right)^{n}(\log x)=\Phi^{-}(h, \log x) .
$$

By Proposition [2.10, $\phi(x)$ is a derivation from $V$ to $V \otimes(\mathbb{C}((x))[\log x],-d / d x)$. Then $E^{+}(-h, x) x^{h(0)}=e^{\phi(x)}$ is an endomorphism from $V$ to $V \otimes \mathbb{C}((x)) \subset V \otimes \mathbb{C}((x))[\log x]$. By Proposition 2.14 we have $E^{+}(-h, x) x^{h(0)} \in \operatorname{PEnd}^{-}(V)$.

\section{$3 \quad$ Differential bialgebra $B(V)$}

In this section we associate a canonical associative algebra $B(V)$ to each nonlocal vertex algebra $V$ and we prove that $B(V)$ has a natural bialgebra structure if $V$ satisfies a certain condition.

First we formulate the following notion: 
Definition 3.1. Let $V$ be a nonlocal vertex algebra. A subset $U$ of $\operatorname{Hom}(V, V \otimes \mathbb{C}((x)))$ is said to be $\Delta$-closed if for any $a(x) \in U$, there exist $a_{(1 i)}(x), a_{(2 i)}(x) \in U$ for $i=1, \ldots, r$ such that

$$
a\left(x_{1}\right) Y\left(v, x_{2}\right)=\sum_{i=1}^{r} Y\left(a_{(1 i)}\left(x_{1}-x_{2}\right) v, x_{2}\right) a_{(2 i)}\left(x_{1}\right) \quad \text { for all } v \in V
$$

In terms of this notion, for any $a(x) \in \mathrm{PDer}^{-}(V),\{a(x), 1\}$ is $\Delta$-closed and for $\psi(x) \in$ PEnd $^{-}(V),\{\psi(x)\}$ is $\Delta$-closed.

Note that the space $\operatorname{Hom}_{\mathbb{C}}(V, V \otimes \mathbb{C}((x)))$, which can be naturally identified with $\operatorname{End}_{\mathbb{C}((x))}(V \otimes \mathbb{C}((x)))$, is an associative algebra over $\mathbb{C}((x))$ with the formal differential operator $\partial=\frac{d}{d x}$ as a $\mathbb{C}$-linear derivation.

The followings are straightforward consequences:

Lemma 3.2. Let $V$ be a nonlocal vertex algebra. a) The linear span of any $\Delta$-closed subset of $\operatorname{Hom}(V, V \otimes \mathbb{C}((x)))$ is $\Delta$-closed. b) The sum of $\Delta$-closed subspaces of $\operatorname{Hom}(V, V \otimes$ $\mathbb{C}((x)))$ is $\Delta$-closed. c) If $U$ is a $\Delta$-closed subset of $\operatorname{Hom}(V, V \otimes \mathbb{C}((x)))$, then $U \cdot U$ (the linear span of $a(x) b(x)$ for $a(x), b(x) \in U)$ is $\Delta$-closed. d) If $U$ is a $\Delta$-closed subspace, $U+\partial(U)$ is $\Delta$-closed.

Definition 3.3. Let $V$ be a nonlocal vertex algebra. Define $B(V)$ to be the sum of all the $\Delta$-closed subspaces $U$ of $\operatorname{Hom}(V, V \otimes \mathbb{C}((x)))$ such that

$$
a(x) \mathbf{1} \in \mathbb{C} \mathbf{1} \quad \text { for } a(x) \in U \text {. }
$$

Proposition 3.4. For any nonlocal vertex algebra $V, B(V)$ is a $\Delta$-closed associative subalgebra of $\operatorname{Hom}(V, V \otimes \mathbb{C}((x)))$ and it is closed under the derivation $\partial=\frac{d}{d x}$. Furthermore, $V$ is a module for $(B(V), \partial)$ with $Y_{V}\left(a(x), x_{0}\right)=a\left(x_{0}\right)$ for $a(x) \in B(V)$.

Proof. From Lemma 3.2 b), $B(V)$ is $\Delta$-closed, and then by Lemma 3.2 c), $B(V) \cdot B(V)$ is $\Delta$-closed. Clearly, we have $B(V) \mathbf{1} \subset \mathbb{C} \mathbf{1}$. Then $B(V) B(V) \mathbf{1} \subset \mathbb{C} \mathbf{1}$. Now, it follows that $B(V) \cdot B(V) \subset B(V)$. This proves that $B(V)$ is a subalgebra. By Lemma 3.2 d), $B(V)+\partial B(V)$ is $\Delta$-closed and we have $\partial B(V) \mathbf{1}=0$. Then $\partial B(V) \subset B(V)$.

Next, we prove that $V$ is a module for $B(V)$ viewed as a nonlocal vertex algebra. First, by definition we have

$$
Y_{V}\left(a(x), x_{0}\right) v=a\left(x_{0}\right) v \in V \otimes \mathbb{C}\left(\left(x_{0}\right)\right) \subset V\left(\left(x_{0}\right)\right) \quad \text { for } a(x) \in B(V), v \in V .
$$

For $a(x), b(x) \in B(V), v \in V$, we have

$$
Y_{V}\left(a(x), x_{0}+x_{2}\right) Y_{V}\left(b(x), x_{2}\right) v=a\left(x_{0}+x_{2}\right) b\left(x_{2}\right) v
$$

and

$$
Y_{V}\left(Y\left(a(x), x_{0}\right) b(x), x_{2}\right) v=\left.\left(a\left(x+x_{0}\right) b(x) v\right)\right|_{x=x_{2}}=a\left(x_{2}+x_{0}\right) b\left(x_{2}\right) v
$$


noticing that $Y\left(a(x), x_{0}\right) b(x)=\left(e^{x_{0} \frac{d}{d x}} a(x)\right) b(x)=a\left(x+x_{0}\right) b(x)$. Since $b\left(x_{2}\right) v \in V \otimes$ $\mathbb{C}\left(\left(\left(x_{2}\right)\right)\right.$, there exists $l \in \mathbb{N}$ such that $x_{1}^{l} a\left(x_{1}\right) b\left(x_{2}\right) v \in V \otimes \mathbb{C}\left(\left(x_{2}\right)\right)\left[\left[x_{1}\right]\right]$. Then

$$
\left(x_{0}+x_{2}\right)^{l} a\left(x_{0}+x_{2}\right) b\left(x_{2}\right) v=\left(x_{2}+x_{0}\right)^{l} a\left(x_{2}+x_{0}\right) b\left(x_{2}\right) v .
$$

Consequently, we have

$$
\left(x_{0}+x_{2}\right)^{l} Y\left(a(x), x_{0}+x_{2}\right) Y\left(b(x), x_{2}\right) v=\left(x_{0}+x_{2}\right)^{l} Y\left(Y\left(a(x), x_{0}\right) b(x), x_{2}\right) v .
$$

We also have $Y_{V}(1, x)=1_{V}$. Thus $V$ is a module for $B(V)$ viewed as a nonlocal vertex algebra.

Remark 3.5. Recall that a coalgebra over $\mathbb{C}$ is a vector space $A$ equipped with two linear maps $\Delta: A \rightarrow A \otimes A$, called the comultiplication map, and $\varepsilon: A \rightarrow \mathbb{C}$, called the counit map, such that

$$
\begin{aligned}
& (1 \otimes \Delta) \Delta=(\Delta \otimes 1) \Delta \\
& \sum \varepsilon\left(a_{(1)}\right) a_{(2)}=\sum \varepsilon\left(a_{(2)}\right) a_{(1)}=a \quad \text { for } a \in A .
\end{aligned}
$$

A bialgebra is a (unital) associative algebra equipped with a coalgebra structure such that the comultiplication map and the counit map are homomorphisms of associative algebras.

For convenience, we formulate the following notion:

Definition 3.6. A differential bialgebra is a bialgebra $(B, \Delta, \varepsilon)$ equipped with a derivation $\partial$ such that $\varepsilon \circ \partial=0$ and $\Delta \partial=(\partial \otimes 1+1 \otimes \partial) \Delta$.

We shall prove that if a nonlocal vertex algebra $V$ satisfies a certain condition, $B(V)$ has a natural bialgebra structure. Recall from [EK that a nonlocal vertex algebra $V$ is nondegenerate if for any positive integer $n$, the linear map

$$
Z_{n}: V^{\otimes n} \otimes \mathbb{C}\left(\left(x_{1}\right)\right) \cdots\left(\left(x_{n}\right)\right) \rightarrow V\left(\left(x_{1}\right)\right) \cdots\left(\left(x_{n}\right)\right)
$$

defined by

$$
Z_{n}\left(v_{(1)} \otimes \cdots \otimes v_{(n)} \otimes f\right)=f Y\left(v_{(1)}, x_{1}\right) \cdots Y\left(v_{(n)}, x_{n}\right) \mathbf{1}
$$

is injective.

Remark 3.7. Let $V$ be a nonlocal vertex algebra. For any positive integer $n$, we define a linear map

$$
X_{n}: V^{(n+1)} \otimes \mathbb{C}\left(\left(x_{1}\right)\right) \cdots\left(\left(x_{n}\right)\right) \rightarrow V\left(\left(x_{1}\right)\right) \cdots\left(\left(x_{n}\right)\right)
$$

by

$$
X_{n}\left(v_{(1)} \otimes \cdots \otimes v_{(n)} \otimes v \otimes f\right)=f Y\left(v_{(1)}, x_{1}\right) \cdots Y\left(v_{(n)}, x_{n}\right) v
$$

It was proved in [Li4] that if $Z_{n+1}$ is injective, so is $X_{n}$. Thus if $V$ is nondegenerate, for every positive integer $n, X_{n}$ is injective, in particular, $X_{1}$ and $X_{2}$ are injective. 
Theorem 3.8. Let $V$ be a nondegenerate nonlocal vertex algebra. Then $B(V)$ is a differential bialgebra with the comultiplication $\Delta$ and the counit $\varepsilon$ uniquely determined by

$$
\begin{aligned}
& a(x) \mathbf{1}=\varepsilon(a(x)) \mathbf{1}, \\
& \Delta(a(x))=\sum a_{(1)}(x) \otimes a_{(2)}(x)
\end{aligned}
$$

for $a(x) \in B(V)$, where $a\left(x_{1}\right) Y\left(v, x_{2}\right)=\sum Y\left(a_{(1)}\left(x_{1}-x_{2}\right) v, x_{2}\right) a_{(2)}\left(x_{1}\right)$ for all $v \in V$ and we are using the coalgebra Sigma notation. Furthermore, we have

$$
\begin{aligned}
& \operatorname{PDer}^{-}(V)=\{a \in B(V) \mid \Delta(a)=a \otimes 1+1 \otimes a\}, \\
& \operatorname{PEnd}^{-}(V)=\{a \in B(V) \mid \Delta(a)=a \otimes a\} .
\end{aligned}
$$

Proof. First we show that $\Delta$ is well defined. It suffices to prove that if

$$
0 \neq \sum_{r=1}^{k} a_{r}(x) \otimes b_{r}(x) \in B(V) \otimes B(V)
$$

then there exist $u, v \in V$ such that

$$
\sum_{r=1}^{k} Y\left(a_{r}\left(x_{1}-x_{2}\right) u, x_{2}\right) b_{r}\left(x_{1}\right) v \neq 0
$$

From (3.9), there exist $u, v \in V$ such that

$0 \neq \sum_{r=1}^{k} a_{r}\left(-x_{2}\right) u \otimes b_{r}\left(x_{1}\right) v \in\left(V \otimes \mathbb{C}\left(\left(x_{2}\right)\right)\right) \otimes\left(V \otimes \mathbb{C}\left(\left(x_{1}\right)\right)\right) \subset\left(V \otimes V \otimes \mathbb{C}\left(\left(x_{2}\right)\right)\right)\left(\left(x_{1}\right)\right)$ which (by applying $e^{-x_{1} \partial / \partial x_{2}}$ ) implies

$$
0 \neq \sum_{r=1}^{k} a_{r}\left(-x_{2}+x_{1}\right) u \otimes b_{r}\left(x_{1}\right) v \in\left(V \otimes V \otimes \mathbb{C}\left(\left(x_{2}\right)\right)\right)\left(\left(x_{1}\right)\right) .
$$

Since the linear map $X_{1}$ is injective, we have

$$
0 \neq \sum_{r=1}^{k} Y\left(a_{r}\left(-x_{2}+x_{1}\right) u, x_{2}\right) b_{r}\left(x_{1}\right) v \in V\left(\left(x_{2}\right)\right)\left(\left(x_{1}\right)\right) \text {. }
$$

Let $l \in \mathbb{N}$ be such that $a_{r}(x) u \in V \otimes \mathbb{C}[[x]]$ for $r=1, \ldots, k$. Consequently,

$$
\left(x_{1}-x_{2}\right)^{l} a_{r}\left(x_{1}-x_{2}\right) u=\left(x_{1}-x_{2}\right)^{l} a_{r}\left(-x_{2}+x_{1}\right) u .
$$

Multiplying (3.11) by $\left(x_{1}-x_{2}\right)^{l}$ and then using cancellation we get (3.10). This proves that $\Delta$ is well defined. 
Next we establish the coassociativity. Let $a(x) \in B(V)$ and let $u, v, w \in V$. We have

$$
\begin{aligned}
& a\left(x_{1}\right) Y\left(u, x_{0}+x_{2}\right) Y\left(v, x_{2}\right) w \\
= & \sum Y\left(a_{(1)}\left(x_{1}-x_{0}-x_{2}\right) u, x_{0}+x_{2}\right) a_{(2)}\left(x_{1}\right) Y\left(v, x_{2}\right) w \\
= & \sum Y\left(a_{(1)}\left(x_{1}-x_{0}-x_{2}\right) u, x_{0}+x_{2}\right) Y\left(a_{(2,1)}\left(x_{1}-x_{2}\right) v, x_{2}\right) a_{(2,2)}\left(x_{1}\right) w .
\end{aligned}
$$

On the other hand, we have

$$
\begin{aligned}
& a\left(x_{1}\right) Y\left(Y\left(u, x_{0}\right) v, x_{2}\right) w \\
= & \sum Y\left(a_{(1)}\left(x_{1}-x_{2}\right) Y\left(u, x_{0}\right) v, x_{2}\right) a_{(2)}\left(x_{1}\right) w \\
= & \sum Y\left(Y\left(a_{(1,1)}\left(x_{1}-x_{2}-x_{0}\right) u, x_{0}\right) a_{(1,2)}\left(x_{1}-x_{2}\right) v, x_{2}\right) a_{(2)}\left(x_{1}\right) w .
\end{aligned}
$$

With $a_{(1,1)}(x) u, a_{(1,2)}(x) v, a_{(2)}(x) w \in V \otimes \mathbb{C}((x))$, there exists $l \in \mathbb{N}$ such that

$$
\begin{aligned}
& \left(x_{0}+x_{2}\right)^{l} Y\left(Y\left(u, x_{0}\right) v, x_{2}\right) w=Y\left(u, x_{0}+x_{2}\right) Y\left(v, x_{2}\right) w \\
& \left(x_{0}+x_{2}\right)^{l} \sum Y\left(Y\left(a_{(1,1)}\left(x_{1}-x_{2}-x_{0}\right) u, x_{0}\right) a_{(1,2)}\left(x_{1}-x_{2}\right) v, x_{2}\right) a_{(2)}\left(x_{1}\right) w \\
& \quad=\left(x_{0}+x_{2}\right)^{l} \sum Y\left(a_{(1,1)}\left(x_{1}-x_{2}-x_{0}\right) u, x_{0}+x_{2}\right) Y\left(a_{(1,2)}\left(x_{1}-x_{2}\right) v, x_{2}\right) a_{(2)}\left(x_{1}\right) w
\end{aligned}
$$

Then using (3.12) and (3.13) we obtain

$$
\begin{aligned}
& \sum\left(x_{0}+x_{2}\right)^{l} Y\left(a_{(1)}\left(x_{1}-x_{0}-x_{2}\right) u, x_{0}+x_{2}\right) Y\left(a_{(2,1)}\left(x_{1}-x_{2}\right) v, x_{2}\right) a_{(2,2)}\left(x_{1}\right) w \\
= & \sum\left(x_{0}+x_{2}\right)^{l} Y\left(a_{(1,1)}\left(x_{1}-x_{2}-x_{0}\right) u, x_{0}+x_{2}\right) Y\left(a_{(1,2)}\left(x_{1}-x_{2}\right) v, x_{2}\right) a_{(2)}\left(x_{1}\right) w
\end{aligned}
$$

which by cancellation and substitution yields

$$
\begin{aligned}
& \sum Y\left(a_{(1)}\left(x_{1}-x_{3}\right) u, x_{3}\right) Y\left(a_{(2,1)}\left(x_{1}-x_{2}\right) v, x_{2}\right) a_{(2,2)}\left(x_{1}\right) w \\
= & \sum Y\left(a_{(1,1)}\left(x_{1}-x_{3}\right) u, x_{3}\right) Y\left(a_{(1,2)}\left(x_{1}-x_{2}\right) v, x_{2}\right) a_{(2)}\left(x_{1}\right) w .
\end{aligned}
$$

By multiplying by certain nonnegative powers of $\left(x_{1}-x_{3}\right)$ and $\left(x_{2}-x_{3}\right)$ and then using cancellation we get

$$
\begin{aligned}
& \sum Y\left(a_{(1)}\left(-x_{3}+x_{1}\right) u, x_{3}\right) Y\left(a_{(2,1)}\left(-x_{2}+x_{1}\right) v, x_{2}\right) a_{(2,2)}\left(x_{1}\right) w \\
= & \sum Y\left(a_{(1,1)}\left(-x_{3}+x_{1}\right) u, x_{3}\right) Y\left(a_{(1,2)}\left(-x_{2}+x_{1}\right) v, x_{2}\right) a_{(2)}\left(x_{1}\right) w .
\end{aligned}
$$

Since $X_{2}$ is injective, we have

$$
\sum a_{(1)}\left(x_{3}\right) u \otimes a_{(2,1)}\left(x_{2}\right) v \otimes a_{(2,2)}\left(x_{1}\right) w=\sum a_{(1,1)}\left(x_{3}\right) u \otimes a_{(1,2)}\left(x_{2}\right) \otimes a_{(2)}\left(x_{1}\right) w .
$$

This gives the coassociativity. It is straightforward to show that $\Delta$ is an algebra homomorphism. 
As $a(x) \mathbf{1} \in \mathbb{C} \mathbf{1}$ for $a(x) \in B(V)$ by assumption, the map $\varepsilon: B(V) \rightarrow \mathbb{C}$ is well defined. It is straightforward to see that $\varepsilon$ is an algebra homomorphism. For $a(x) \in B(V)$, we have

$$
\begin{aligned}
a\left(x_{1}\right) & =a\left(x_{1}\right) Y\left(\mathbf{1}, x_{2}\right) \\
& =\sum Y\left(a_{(1)}\left(x_{1}-x_{2}\right) \mathbf{1}, x_{2}\right) a_{(2)}\left(x_{1}\right) \\
& =\sum Y\left(\varepsilon\left(a_{(1)}(x)\right) \mathbf{1}, x_{2}\right) a_{(2)}\left(x_{1}\right) \\
& =\sum \varepsilon\left(a_{(1)}(x)\right) a_{(2)}\left(x_{1}\right)
\end{aligned}
$$

and

$$
\begin{aligned}
a(x) v & =\operatorname{Res}_{x_{2}} x_{2}^{-1} a\left(x_{1}\right) Y\left(v, x_{2}\right) \mathbf{1} \\
& =\sum \operatorname{Res}_{x_{2}} x_{2}^{-1} Y\left(a_{(1)}\left(x_{1}-x_{2}\right) v, x_{2}\right) a_{(2)}\left(x_{1}\right) \mathbf{1} \\
& =\sum \operatorname{Res}_{x_{2}} x_{2}^{-1} \varepsilon\left(a_{(2)}(x)\right) Y\left(a_{(1)}\left(x_{1}-x_{2}\right) v, x_{2}\right) \mathbf{1} \\
& =\sum \operatorname{Res}_{x_{2}} x_{2}^{-1} \varepsilon\left(a_{(2)}(x)\right) e^{-x_{2} \frac{\partial}{\partial x_{1}}} Y\left(a_{(1)}\left(x_{1}\right) v, x_{2}\right) \mathbf{1} \\
& =\sum \varepsilon\left(a_{(2)}(x)\right) a_{(1)}\left(x_{1}\right) v .
\end{aligned}
$$

This proves that $\varepsilon$ is a counit. Therefore, $(B(V), \Delta, \varepsilon)$ is a bialgebra.

It is straightforward to show

$$
\Delta \partial=(\partial \otimes 1+1 \otimes \partial) \Delta, \quad \varepsilon \circ \partial=0 .
$$

Thus $B(V)$ is a differential bialgebra. The assertions (3.7) and (3.8) are clear.

We have the following consequence:

Corollary 3.9. Let $V$ be a nonlocal vertex algebra. Assume that $V$ is of countable dimension over $\mathbb{C}$ and that $V$ as a $V$-module is irreducible. Then all the assertions of Theorem 3.8 hold. In particular, if $V$ is a simple vertex operator algebra in the sense of [FLM], all the assertions of Theorem 3.8 hold.

Proof. Under the assumptions, by [Li4] and [Li6], $V$ is nondegenerate. Then it follows immediately from Theorem 3.8 .

Proposition 3.10. Let $V$ be a nondegenerate ordinary vertex algebra and let $B(V)^{c}$ be the sum of all the $\Delta$-closed subspaces $U$ of $\operatorname{Hom}(V, V \otimes \mathbb{C}((x)))$ such that

$$
\begin{aligned}
& a(x) \mathbf{1} \in \mathbb{C} \mathbf{1}, \\
& {[\mathcal{D}, a(x)]=\frac{d}{d x} a(x) \quad \text { for } a(x) \in U .}
\end{aligned}
$$

Then $B(V)^{c}$ is a cocommutative sub-bialgebra of $B(V)$. 
Proof. It is straightforward to show that $B(V)^{c}$ is a sub-differential bialgebra of $B(V)$. Now we prove the cocommutativity. Let $a(x) \in B(V)^{c}$ with $\Delta(a(x))=\sum a_{(1)}(x) \otimes a_{(2)}(x)$. For $u, v \in V$, we have

$$
a\left(x_{1}\right) Y(u, x) v=\sum Y\left(a_{(1)}\left(x_{1}-x\right) u, x\right) a_{(2)}\left(x_{1}\right) v .
$$

Using the skew symmetry of the vertex algebra $V$ and (3.19) we get

$$
\begin{aligned}
& a\left(x_{1}\right) Y(u, x) v=a\left(x_{1}\right) e^{x \mathcal{D}} Y(v,-x) u=e^{x \mathcal{D}} a\left(x_{1}-x\right) Y(v,-x) u, \\
& \sum Y\left(a_{(1)}\left(x_{1}-x\right) u, x\right) a_{(2)}\left(x_{1}\right) v=\sum e^{x \mathcal{D}} Y\left(a_{(2)}\left(x_{1}\right) v,-x\right) a_{(1)}\left(x_{1}-x\right) u .
\end{aligned}
$$

Consequently,

$$
a\left(x_{1}-x\right) Y(v,-x) u=\sum Y\left(a_{(2)}\left(x_{1}\right) v,-x\right) a_{(1)}\left(x_{1}-x\right) u
$$

which is,

$$
a\left(x_{1}\right) Y(v, x) u=\sum Y\left(a_{(2)}\left(x_{1}-x\right) v, x\right) a_{(1)}\left(x_{1}\right) u .
$$

Thus $\Delta(a(x))=\sum a_{(2)}(x) \otimes a_{(1)}(x)$. This proves the cocommutativity.

\section{Smash product nonlocal vertex algebras}

In this section we formulate a notion of vertex bialgebra and a notion of module nonlocal vertex algebra for a vertex bialgebra. We then give a smash product construction of nonlocal vertex algebras.

The following is our notion of vertex bialgebra:

Definition 4.1. A vertex bialgebra is a nonlocal vertex algebra $V$ equipped with a coalgebra structure $(V, \Delta, \varepsilon)$ such that $\Delta: V \rightarrow V \otimes V$ and $\varepsilon: V \rightarrow \mathbb{C}$ are homomorphisms of nonlocal vertex algebras.

Example 4.2. Here we extend Borcherds' construction of vertex algebras for vertex bialgebras. Let $(B, \Delta, \varepsilon, \partial)$ be a differential bialgebra (see Definition 3.6). First we have a nonlocal vertex algebra $(B, \partial)$. From definition we have $\varepsilon(1)=1$ and $\Delta(1)=1 \otimes 1$. Furthermore, for $a, b \in B$ we have

$$
\begin{aligned}
& \varepsilon(Y(a, x) b)=\varepsilon\left(\left(e^{x \partial} a\right) b\right)=\varepsilon\left(e^{x \partial} a\right) \varepsilon(b)=\varepsilon(a) \varepsilon(b), \\
& \Delta(Y(a, x) b)=\Delta\left(\left(e^{x \partial} a\right) b\right)=\Delta\left(e^{x \partial} a\right) \Delta(b)=\left(e^{x(\partial \otimes 1+1 \otimes \partial)} \Delta(a)\right) \Delta(b)=Y(\Delta(a), x) \Delta(b) .
\end{aligned}
$$

This shows that $\Delta$ and $\varepsilon$ are homomorphisms of nonlocal vertex algebras. Therefore, $B$ is a vertex bialgebra.

Example 4.3. Let $\mathfrak{g}$ be a Lie algebra equipped with a derivation $d$. Then $U(\mathfrak{g})$ is a Hopf algebra with $d$ naturally extended to a derivation of $U(\mathfrak{g})$. It is straightforward to show that $U(\mathfrak{g})$ viewed as a bialgebra equipped with the derivation $d$ is a differential bialgebra. In view of Example 4.2, $U(\mathfrak{g})$ is naturally a vertex bialgebra. 
Example 4.4. Here we show that vertex algebras associated with vertex Lie algebras can be made into vertex bialgebras. Let $C$ be a vertex Lie algebra (see $[\mathrm{P}]$ ), or namely a conformal Lie algebra (see $[\mathrm{K}]$ ). Associated to $C$ we have an honest Lie algebra $\mathcal{L}_{C}$. As a vector space,

$$
\mathcal{L}_{C}=\left(C \otimes \mathbb{C}\left[t, t^{-1}\right]\right) /(\partial \otimes 1+1 \otimes d / d t)\left(C \otimes \mathbb{C}\left[t, t^{-1}\right]\right)
$$

For $a \in C, n \in \mathbb{Z}$, we denote by $a(n)$ the element of $\mathcal{L}_{C}$ corresponding to $a \otimes t^{n}$. The Lie algebra $\mathcal{L}_{C}$ has a polar decomposition $\mathcal{L}_{C}=\mathcal{L}_{C}^{+} \oplus \mathcal{L}_{C}^{-}$into subalgebras, where $\mathcal{L}_{C}^{+}$is spanned by $a(n)$ for $a \in C, n \geq 0$ and $\mathcal{L}_{C}^{-}$is spanned by $a(n)$ for $a \in C, n<0$. Form the induced module of $\mathcal{L}_{C}$ from the trivial $\mathcal{L}_{C}^{+}$-module $\mathbb{C}$ :

$$
V_{C}=U\left(\mathcal{L}_{C}\right) \otimes_{U\left(\mathcal{L}_{C}^{+}\right)} \mathbb{C}
$$

In view of the P-B-W theorem, $V_{C}=U\left(\mathcal{L}_{C}^{-}\right)$as vector spaces. Set

$$
\mathbf{1}=1 \otimes 1 \in V_{C} .
$$

Identify $C$ as a subspace of $V_{C}$ through the linear map $u \mapsto u(-1) \mathbf{1}$. Then (see [DLM], $[\mathrm{P}])$ there exists a unique vertex algebra structure on $V_{C}$ with $\mathbf{1}$ as the vacuum vector and with

$$
Y(u, x)=u(x)=\sum_{n \in \mathbb{Z}} u(n) x^{-n-1} \quad \text { for } u \in C .
$$

Equip $V_{C} \otimes V_{C}$ with the tensor product $\mathcal{L}_{C}$-module structure. We have $\mathcal{L}_{C}^{+}(\mathbf{1} \otimes \mathbf{1})=0$. Then there exists a unique $\mathcal{L}_{C}$-module map $\Delta$ from $V_{C}$ to $V_{C} \otimes V_{C}$ such that $\Delta(\mathbf{1})=\mathbf{1} \otimes \mathbf{1}$. For $a \in C$, we have

$$
\Delta(a)=\Delta(a(-1) \mathbf{1})=a(-1) \Delta(\mathbf{1})=a(-1) \mathbf{1} \otimes \mathbf{1}+\mathbf{1} \otimes a(-1) \mathbf{1}=a \otimes \mathbf{1}+\mathbf{1} \otimes a .
$$

Then

$$
Y(\Delta(a), x)=Y(a, x) \otimes 1+1 \otimes Y(a, x)=a(x) \otimes 1+1 \otimes a(x) .
$$

Now, for any $a \in C, v \in V_{C}$, we have

$$
\Delta(Y(a, x) v)=\Delta(a(x) v)=a(x) \Delta(v)=(a(x) \otimes 1+1 \otimes a(x)) \Delta(v)=Y(\Delta(a), x) \Delta(v) .
$$

As $C$ generates $V_{C}$ as a vertex algebra, $\Delta$ is a vertex algebra homomorphism. Similarly, equipping $\mathbb{C}$ with the trivial $\mathcal{L}_{C}$-module, we have a unique $\mathcal{L}_{C}$-module map $\varepsilon: V_{C} \rightarrow \mathbb{C}$ such that $\varepsilon(\mathbf{1})=1$. For $a \in C, v \in V_{C}$, as $\varepsilon(a)=\varepsilon(a(-1) \mathbf{1})=a(-1) \varepsilon(\mathbf{1})=0$, we have

$$
\varepsilon(Y(a, x) v)=\varepsilon(a(x) v)=a(x) \varepsilon(v)=0=Y(\varepsilon(a), x) \varepsilon(v) .
$$

From the same reasoning $\varepsilon$ is a vertex algebra homomorphism. Therefore, the associated vertex algebra $V_{C}$ equipped with the maps $\Delta$ and $\varepsilon$ defined above is a vertex bialgebra. [M] :

The following is a vertex analogue of the notion of module algebra for a bialgebra (cf. 
Definition 4.5. Let $H$ be a vertex bialgebra. An $H$-module nonlocal vertex algebra is a nonlocal vertex algebra $V$ equipped with an $H$-module structure such that

$$
\begin{aligned}
& Y(h, x) v \in V \otimes \mathbb{C}((x)), \\
& Y(h, x) \mathbf{1}_{V}=\varepsilon(h) \mathbf{1}_{V} \\
& Y\left(h, x_{1}\right) Y\left(u, x_{2}\right) v=\sum Y\left(Y\left(h_{(1)}, x_{1}-x_{2}\right) u, x_{2}\right) Y\left(h_{(2)}, x_{1}\right) v
\end{aligned}
$$

for $h \in H, u, v \in V$, where $\mathbf{1}_{V}$ denotes the vacuum vector of $V$.

The following result is very useful in constructing module nonlocal vertex algebras:

Lemma 4.6. Let $H$ be a vertex bialgebra and let $V$ be a nonlocal vertex algebra. Suppose that $\left(V, Y_{V}^{H}\right)$ is an $H$-module and $S$ is a generating subset of $H$ as a nonlocal vertex algebra such that for $h \in S, u, v \in V$,

$$
\begin{aligned}
& Y_{V}^{H}(h, x) \in \operatorname{Hom}(V, V \otimes \mathbb{C}((x))), \\
& Y_{V}^{H}(h, x) \mathbf{1}=\varepsilon(h) \mathbf{1}, \\
& \left.Y_{V}^{H}\left(h, x_{1}\right) Y\left(u, x_{2}\right) v=\sum Y\left(Y_{V}^{H}\left(h_{(1)}, x_{1}-x_{2}\right) u, x_{2}\right) Y_{V}^{H}\left(h_{(2)}\right), x_{1}\right) v .
\end{aligned}
$$

Then $V$ equipped with the $H$-module structure $Y_{V}^{H}$ is an $H$-module nonlocal vertex algebra. Proof. Let $g, h \in H$ be such that

$$
Y_{V}^{H}(g, x), \quad Y_{V}^{H}(h, x) \in \operatorname{Hom}(V, V \otimes \mathbb{C}((x))) .
$$

For any $v \in V$, as $\left(V, Y_{V}^{H}\right)$ is an $H$-module, there exists a nonnegative integer $l$ such that

$$
\left(x_{0}+x_{2}\right)^{l} Y_{V}^{H}\left(Y\left(g, x_{0}\right) h, x_{2}\right) v=\left(x_{0}+x_{2}\right)^{l} Y_{V}^{H}\left(g, x_{0}+x_{2}\right) Y_{V}^{H}\left(h, x_{2}\right) v .
$$

Since $Y_{V}^{H}\left(h, x_{2}\right) v \in V \otimes \mathbb{C}\left(\left(x_{2}\right)\right)$, there exists $l^{\prime} \in \mathbb{N}$ such that $x^{l^{\prime}} Y_{V}^{H}(g, x) Y_{V}^{H}\left(h, x_{2}\right) v \in$ $V[[x]] \otimes \mathbb{C}\left(\left(x_{2}\right)\right)$. Then

$$
\left(x_{0}+x_{2}\right)^{l^{\prime}} Y_{V}^{H}\left(g, x_{0}+x_{2}\right) Y_{V}^{H}\left(h, x_{2}\right) v=\left(x_{2}+x_{0}\right)^{l^{\prime}} Y_{V}^{H}\left(g, x_{2}+x_{0}\right) Y_{V}^{H}\left(h, x_{2}\right) v .
$$

Combining this with (4.7) we get

$$
\left(x_{0}+x_{2}\right)^{l+l^{\prime}} Y_{V}^{H}\left(Y\left(g, x_{0}\right) h, x_{2}\right) v=\left(x_{2}+x_{0}\right)^{l+l^{\prime}} Y_{V}^{H}\left(g, x_{2}+x_{0}\right) Y_{V}^{H}\left(h, x_{2}\right) v .
$$

By cancellation we get

$$
Y_{V}^{H}\left(Y\left(g, x_{0}\right) h, x_{2}\right) v=Y_{V}^{H}\left(g, x_{2}+x_{0}\right) Y_{V}^{H}\left(h, x_{2}\right) v=\left.\left(\left(e^{x_{0} \frac{d}{d x}} Y_{V}^{H}(g, x)\right) Y_{V}^{H}(h, x)\right)\right|_{x=x_{2}} v .
$$

Since $S$ generates $H$, we have $Y_{V}^{H}(h, x) \in \operatorname{Hom}(V, V \otimes \mathbb{C}((x)))$ for all $h \in H$. Because $\Delta, \varepsilon$ are homomorphisms of nonlocal vertex algebras and because $S$ generates $H$ as a nonlocal vertex algebra, it follows that (4.5) and (4.6) hold for all $h \in H$. Thus $V$ is an $H$-module nonlocal vertex algebra. 
We have the following simple result:

Lemma 4.7. Let $H$ be a vertex bialgebra and let $V$ be an $H$-module nonlocal vertex algebra with the $H$-module structure $Y_{V}^{H}$. Then $Y_{V}^{H}$ is a homomorphism of nonlocal vertex algebras from $H$ to $B(V)$. In particular,

$$
Y_{V}^{H}\left(Y\left(g, x_{0}\right) h, x\right) v=\left(e^{x_{0} \frac{d}{d x}} Y_{V}^{H}(g, x)\right) Y_{V}^{H}(h, x) v
$$

for $g, h \in H, v \in V$.

Proof. From Definition 4.5, we have $Y_{V}^{H}(h, x) \in \operatorname{Hom}(V, V \otimes \mathbb{C}((x))), Y_{V}^{H}(h, x) \mathbf{1}=\varepsilon(h) \mathbf{1} \in$ $\mathbb{C} 1$ for $h \in H$ and $\left\{Y_{V}^{H}(h, x) \mid h \in H\right\}$ is a $\Delta$-closed subspace. Then the linear map $Y_{V}^{H}$ maps $H$ into $B(V)$. From the proof of Lemma 4.6, the particular assertion holds. It follows that $Y_{V}^{H}$ is a homomorphism of nonlocal vertex algebras from $H$ to $B(V)$.

Recall from Corollary 3.9 that if $V$ is nondegenerate, then $B(V)$ is a differential bialgebra, hence a vertex bialgebra.

Proposition 4.8. Let $V$ be a nondegenerate nonlocal vertex algebra. Then $V$ is a $B(V)$ module nonlocal vertex algebra with $Y\left(a(x), x_{0}\right)=a\left(x_{0}\right)$ for $a(x) \in B(V)$. Furthermore, for any vertex bialgebra $H$, an $H$-module nonlocal vertex algebra structure $Y_{V}^{H}$ amounts to a homomorphism of vertex bialgebras from $H$ to $B(V)$.

Proof. By Proposition 3.4. $V$ is a module for $B(V)$ viewed as a nonlocal vertex algebra. For $a(x) \in B(V), v \in V$, we have

$$
Y\left(a(x), x_{0}\right) v=a\left(x_{0}\right) v \in V \otimes \mathbb{C}\left(\left(x_{0}\right)\right),
$$

and

$$
\begin{aligned}
& Y\left(a(x), x_{0}\right) \mathbf{1}=a\left(x_{0}\right) \mathbf{1}=\varepsilon(a(x)) \mathbf{1}, \\
& Y\left(a(x), x_{1}\right) Y\left(v, x_{2}\right)=a\left(x_{1}\right) Y\left(v, x_{2}\right)=\sum Y\left(a_{(1)}\left(x_{1}-x_{2}\right) v, x_{2}\right) a_{(2)}\left(x_{1}\right) \\
& \quad=\sum Y\left(Y\left(a_{(1)}(x), x_{1}-x_{2}\right) v, x_{2}\right) Y\left(a_{(2)}(x), x_{1}\right) .
\end{aligned}
$$

Therefore $V$ is a $B(V)$-module nonlocal vertex algebra.

Let $H$ be a vertex bialgebra and assume that $\left(V, Y_{V}^{H}\right)$ is an $H$-module nonlocal vertex algebra. From Lemma 4.7. $Y_{V}^{H}$ is a homomorphism of nonlocal vertex algebras from $H$ to $B(V)$. For $h \in H$, since $Y_{V}^{H}(h, x) \mathbf{1}=\varepsilon(h) \mathbf{1}$, by definition we have $\varepsilon(Y(h, x))=\varepsilon(h)$. For $h \in H, v \in V$, we have

$$
Y\left(h, x_{1}\right) Y(v, x)=\sum Y\left(Y\left(h_{(1)}, x_{1}-x\right) v, x\right) Y\left(h_{(2)}, x_{1}\right) .
$$

By definition we have

$$
\Delta(Y(h, x))=\sum Y\left(h_{(1)}, x\right) \otimes Y\left(h_{(2)}, x\right) \in B(V) \otimes B(V) .
$$

Therefore $Y_{V}^{H}$ is a homomorphism of vertex bialgebras.

On the other hand, assume that $Y_{V}^{H}$ is a homomorphism of vertex bialgebras from $H$ to $B(V)$. As $V$ is a $B(V)$-module nonlocal vertex algebra, it is straightforward to see that $V$ equipped with $Y_{V}^{H}$ is an $H$-module nonlocal vertex algebra. 
The following is our smash product construction of nonlocal vertex algebras:

Theorem 4.9. Let $H$ be a vertex bialgebra and let $V$ be an $H$-module nonlocal vertex algebra. Set $V \sharp H=V \otimes H$ as a vector space. For $u, v \in V, h, k \in H$, define

$$
Y^{\sharp}(u \otimes h, x)(v \otimes k)=\sum Y(u, x) Y\left(h_{(1)}, x\right) v \otimes Y\left(h_{(2)}, x\right) k .
$$

Then $V \sharp H$ is a nonlocal vertex algebra with $V$ and $H$ as subalgebras and the following relation holds for $h \in H, u \in V$ :

$$
Y^{\sharp}\left(h, x_{1}\right) Y^{\sharp}\left(u, x_{2}\right)=\sum Y^{\sharp}\left(Y\left(h_{(1)}, x_{1}-x_{2}\right) u, x_{2}\right) Y^{\sharp}\left(h_{(2)}, x_{1}\right) .
$$

Proof. From definition, for $v \in V, h, k \in H$ we have $Y(h, x) v \in V \otimes \mathbb{C}((x))$ and $Y(h, x) k \in$ $H((x))$. It follows that

$$
Y^{\sharp}(u \otimes h, x)(v \otimes k) \in(V \otimes H)((x)) \quad \text { for } u, v \in V, h, k \in H .
$$

As $\Delta(\mathbf{1})=\mathbf{1} \otimes \mathbf{1}$, we have $Y^{\sharp}(\mathbf{1} \otimes \mathbf{1}, x)=1$. Using the counit property of $\varepsilon$ we also have

$$
\begin{aligned}
Y^{\sharp}(u \otimes h, x)(\mathbf{1} \otimes \mathbf{1}) & =\sum Y(u, x) Y\left(h_{(1)}, x\right) \mathbf{1} \otimes Y\left(h_{(2)}, x\right) \mathbf{1} \\
& =\sum \varepsilon\left(h_{(1)}\right) Y(u, x) \mathbf{1} \otimes Y\left(h_{(2)}, x\right) \mathbf{1} \\
& =\sum Y(u, x) \mathbf{1} \otimes Y\left(\varepsilon\left(h_{(1)}\right) h_{(2)}, x\right) \mathbf{1} \\
& =Y(u, x) \mathbf{1} \otimes Y(h, x) \mathbf{1},
\end{aligned}
$$

from which we have

$$
Y^{\sharp}(u \otimes h, x)(\mathbf{1} \otimes \mathbf{1}) \in(V \otimes H)[[x]] \quad \text { and } \quad \lim _{x \rightarrow 0} Y^{\sharp}(u \otimes h, x)(\mathbf{1} \otimes \mathbf{1})=u \otimes h .
$$

Next we prove the weak associativity. Let $u, v, w \in V, g, h, k \in H$. We have

$$
\begin{aligned}
& Y^{\sharp}\left(u \otimes g, x_{0}+x_{2}\right) Y^{\sharp}\left(v \otimes h, x_{2}\right)(w \otimes k) \\
= & \sum Y^{\sharp}\left(u \otimes g, x_{0}+x_{2}\right)\left(Y\left(v, x_{2}\right) Y\left(h_{(1)}, x_{2}\right) w \otimes Y\left(h_{(2)}, x_{2}\right) k\right) \\
= & \sum Y\left(u, x_{0}+x_{2}\right) Y\left(g_{(1)}, x_{0}+x_{2}\right) Y\left(v, x_{2}\right) Y\left(h_{(1)}, x_{2}\right) w \otimes Y\left(g_{(2)}, x_{0}+x_{2}\right) Y\left(h_{(2)}, x_{2}\right) k \\
= & \sum Y\left(u, x_{0}+x_{2}\right) Y\left(Y\left(g_{(1,1)}, x_{0}\right) v, x_{2}\right) Y\left(g_{(1,2)}, x_{0}+x_{2}\right) Y\left(h_{(1)}, x_{2}\right) w \\
& \otimes Y\left(g_{(2)}, x_{0}+x_{2}\right) Y\left(h_{(2)}, x_{2}\right) k .
\end{aligned}
$$

Notice that $Y\left(g_{(1,2)}, x_{0}+x_{2}\right) Y\left(h_{(1)}, x_{2}\right) w \in V \otimes \mathbb{C}\left(\left(x_{0}+x_{2}\right)\right) \otimes \mathbb{C}\left(\left(x_{2}\right)\right)$ and $Y\left(g_{(1,1)}, x_{0}\right) v \in$ $V \otimes \mathbb{C}\left(\left(x_{0}\right)\right)$. Then there exists $l \in \mathbb{N}$ such that

$$
\begin{aligned}
& \left(x_{0}+x_{2}\right)^{l} \sum Y\left(u, x_{0}+x_{2}\right) Y\left(Y\left(g_{(1,1)}, x_{0}\right) v, x_{2}\right) Y\left(g_{(1,2)}, x_{0}+x_{2}\right) Y\left(h_{(1)}, x_{2}\right) w \\
= & \left(x_{0}+x_{2}\right)^{l} \sum Y\left(Y\left(u, x_{0}\right) Y\left(g_{(1,1)}, x_{0}\right) v, x_{2}\right) Y\left(g_{(1,2)}, x_{0}+x_{2}\right) Y\left(h_{(1)}, x_{2}\right) w .
\end{aligned}
$$


Certainly, if necessary we can replace $l$ with a large one so that we also have

$$
\begin{aligned}
& \left(x_{0}+x_{2}\right)^{l} Y\left(g_{(1,2)}, x_{0}+x_{2}\right) Y\left(h_{(1)}, x_{2}\right) w=\left(x_{0}+x_{2}\right)^{l} Y\left(Y\left(g_{(1,2)}, x_{0}\right) h_{(1)}, x_{2}\right) w, \\
& \left(x_{0}+x_{2}\right)^{l} Y\left(g_{(2)}, x_{0}+x_{2}\right) Y\left(h_{(2)}, x_{2}\right) k=\left(x_{0}+x_{2}\right)^{l} Y\left(Y\left(g_{(2)}, x_{0}\right) h_{(2)}, x_{2}\right) k .
\end{aligned}
$$

Then we obtain

$$
\begin{aligned}
& \left(x_{0}+x_{2}\right)^{l} Y^{\sharp}\left(u \otimes g, x_{0}+x_{2}\right) Y^{\sharp}\left(v \otimes h, x_{2}\right)(w \otimes k) \\
= & \left(x_{0}+x_{2}\right)^{l} \sum Y\left(Y\left(u, x_{0}\right) Y\left(g_{(1,1)}, x_{0}\right) v, x_{2}\right) Y\left(Y\left(g_{(1,2)}, x_{0}\right) h_{(1)}, x_{2}\right) w \\
& \otimes Y\left(Y\left(g_{(2)}, x_{0}\right) h_{(2)}, x_{2}\right) k .
\end{aligned}
$$

On the other hand, we have

$$
\begin{aligned}
& Y^{\sharp}\left(Y^{\sharp}\left(u \otimes g, x_{0}\right)(v \otimes h), x_{2}\right)(w \otimes k) \\
= & \sum Y^{\sharp}\left(Y\left(u, x_{0}\right) Y\left(g_{(1)}, x_{0}\right) v \otimes Y\left(g_{(2)}, x_{0}\right) h, x_{2}\right)(w \otimes k) \\
= & \sum Y\left(Y\left(u, x_{0}\right) Y\left(g_{(1)}, x_{0}\right) v, x_{2}\right) Y\left(Y\left(g_{(2,1)}, x_{0}\right) h_{(1)}, x_{2}\right) w \otimes Y\left(Y\left(g_{(2,2)}, x_{0}\right) h_{(2)}, x_{2}\right) k,
\end{aligned}
$$

where we are using the property of $\Delta$ being a homomorphism of nonlocal vertex algebras, which gives

$$
\Delta\left(Y\left(g_{(2)}, x\right) h\right)=Y\left(\Delta\left(g_{(2)}\right), x\right) \Delta(h)=\sum Y\left(g_{(2,1)}, x\right) h_{(1)} \otimes Y\left(g_{(2,2)}, x\right) h_{(2)} .
$$

By the coassociativity of $\Delta$ we have

$$
\sum g_{(1,1)} \otimes g_{(1,2)} \otimes h_{(1)} \otimes g_{(2)} \otimes h_{(2)}=\sum g_{(1)} \otimes g_{(2,1)} \otimes h_{(1)} \otimes g_{(2,2)} \otimes h_{(2)} .
$$

Putting everything together we get

$$
\begin{aligned}
& \left(x_{0}+x_{2}\right)^{l} Y^{\sharp}\left(u \otimes g, x_{0}+x_{2}\right) Y^{\sharp}\left(v \otimes h, x_{2}\right)(w \otimes k) \\
= & \left(x_{0}+x_{2}\right)^{l} Y^{\sharp}\left(Y^{\sharp}\left(u \otimes g, x_{0}\right)(v \otimes h), x_{2}\right)(w \otimes k) .
\end{aligned}
$$

This proves the weak associativity. Thus $V \sharp H$ is a nonlocal vertex algebra.

Clearly, $V$, identified with $V \otimes \mathbb{C}$, is a subalgebra of $V \sharp H$. For $g, h \in H$, we have

$Y^{\sharp}(g, x)(\mathbf{1} \otimes h)=\sum Y\left(g_{(1)}, x\right) \mathbf{1} \otimes Y\left(g_{(2)}, x\right) h=\sum \varepsilon\left(g_{(1)}\right)\left(\mathbf{1} \otimes Y\left(g_{(2)}, x\right) h\right)=\mathbf{1} \otimes Y(g, x) h$ as $g=\sum \varepsilon\left(g_{(1)}\right) g_{(2)}$. Thus $H$, identified with $\mathbb{C} \otimes H$, is a subalgebra.

For $u, v \in V, h, k \in H$, we have

$$
\begin{aligned}
& Y^{\sharp}\left(h, x_{1}\right) Y^{\sharp}\left(u, x_{2}\right)(v \otimes k) \\
= & Y^{\sharp}\left(h, x_{1}\right)\left(Y\left(u, x_{2}\right) v \otimes k\right) \\
= & \sum Y\left(h_{(1)}, x_{1}\right) Y\left(u, x_{2}\right) v \otimes Y\left(h_{(2)}, x_{1}\right) k \\
= & \sum Y\left(Y\left(h_{(1,1)}, x_{1}-x_{2}\right) u, x_{2}\right) Y\left(h_{(1,2)}, x_{1}\right) v \otimes Y\left(h_{(2)}, x_{1}\right) k
\end{aligned}
$$


and

$$
\begin{aligned}
& \sum Y^{\sharp}\left(Y\left(h_{(1)}, x_{1}-x_{2}\right) u, x_{2}\right) Y^{\sharp}\left(h_{(2)}, x_{1}\right)(v \otimes k) \\
= & \sum Y\left(Y\left(h_{(1,1)}, x_{1}-x_{2}\right) u, x_{2}\right) Y\left(h_{(2,1)}, x_{1}\right) v \otimes Y\left(h_{(2,2)}, x_{1}\right) k .
\end{aligned}
$$

As $(\Delta \otimes 1) \Delta(h)=(1 \otimes \Delta) \Delta(h)$, we have

$$
\sum h_{(1,1)} \otimes h_{(1,2)} \otimes h_{(2)}=\sum h_{(1)} \otimes h_{(2,1)} \otimes h_{(2,2)} .
$$

Therefore

$$
Y^{\sharp}\left(h, x_{1}\right) Y^{\sharp}\left(u, x_{2}\right)(v \otimes k)=\sum Y^{\sharp}\left(Y\left(h_{(1)}, x_{1}-x_{2}\right) u, x_{2}\right) Y^{\sharp}\left(h_{(2)}, x_{1}\right)(v \otimes k) .
$$

Now the proof is complete.

Example 4.10. Let $\mathfrak{g}$ be a Lie algebra equipped with a derivation $d$. Recall from Example 4.3 that the universal enveloping algebra $U(\mathfrak{g})$ is naturally a differential bialgebra and hence a vertex bialgebra. Let $V$ be a nonlocal vertex algebra and let $\psi: \mathfrak{g} \rightarrow \operatorname{PDer}^{-}(V)$ be a homomorphism of differential Lie algebras. The map $\psi$ extends uniquely to a homomorphism $\bar{\psi}: U(\mathfrak{g}) \rightarrow B(V)$ of differential algebras. This makes $V$ a $(U(\mathfrak{g}), d)$-module. By Lemma 4.6, $V$ is a $U(\mathfrak{g})$-module nonlocal vertex algebra. Therefore we have the smash product nonlocal vertex algebra $V \sharp U(\mathfrak{g})$.

The following result gives a construction of $V \sharp H$-modules:

Proposition 4.11. Let $H$ be a vertex bialgebra and let $V$ be an $H$-module nonlocal vertex algebra. Suppose that $W$ is a $V$-module and an $H$-module such that

$$
\begin{aligned}
& Y(h, x) w \in W \otimes \mathbb{C}((x)), \\
& Y\left(h, x_{1}\right) Y\left(v, x_{2}\right) w=\sum Y\left(Y\left(h_{(1)}, x_{1}-x_{2}\right) v, x_{2}\right) Y\left(h_{(2)}, x_{1}\right) w
\end{aligned}
$$

for $h \in S, v \in V, w \in W$, where $S$ is a generating subset of $H$ as a nonlocal vertex algebra. Then $W$ is a module for $V \sharp H$ with

$$
Y_{W}(v \otimes h, x) w=Y(v, x) Y(h, x) w
$$

for $h \in H, v \in V, w \in W$.

Proof. The proof of Lemma 4.6 shows that (4.12) and (4.13) hold for all $h \in H$. For $v \in V, h \in H, w \in W$, we have

$$
Y_{W}(v \otimes h, x) w=Y(v, x) Y(h, x) w \in W((x))
$$

as $Y(h, x) w \in W \otimes \mathbb{C}((x))$ and $Y(v, x) \in \operatorname{Hom}(W, W((x)))$. From definition we have $Y_{W}(\mathbf{1} \otimes 1, x)=Y(\mathbf{1}, x) Y(1, x)=1$. Let $u, v \in V, g, h \in H, w \in W$. We have

$$
\begin{aligned}
& Y_{W}\left(u \otimes g, x_{0}+x_{2}\right) Y_{W}\left(v \otimes h, x_{2}\right) w \\
= & Y\left(u, x_{0}+x_{2}\right) Y\left(g, x_{0}+x_{2}\right) Y\left(v, x_{2}\right) Y\left(h, x_{2}\right) w \\
= & \sum Y\left(u, x_{0}+x_{2}\right) Y\left(Y\left(g_{(1)}, x_{0}\right) v, x_{2}\right) Y\left(g_{(2)}, x_{0}+x_{2}\right) Y\left(h, x_{2}\right) w .
\end{aligned}
$$


Notice that

$$
Y\left(g_{(2)}, x_{0}+x_{2}\right) Y\left(h, x_{2}\right) w \in W \otimes \mathbb{C}\left(\left(x_{2},\left(x_{0}+x_{2}\right)\right)\right), \quad Y\left(g_{(1)}, x_{0}\right) v \in V \otimes \mathbb{C}\left(\left(x_{0}\right)\right) .
$$

Then there exists a nonnegative integer $l$ such that

$$
\begin{aligned}
& \sum\left(x_{0}+x_{2}\right)^{l} Y\left(u, x_{0}+x_{2}\right) Y\left(Y\left(g_{(1)}, x_{0}\right) v, x_{2}\right) Y\left(g_{(2)}, x_{0}+x_{2}\right) Y\left(h, x_{2}\right) w \\
= & \sum\left(x_{0}+x_{2}\right)^{l} Y\left(Y\left(u, x_{0}\right) Y\left(g_{(1)}, x_{0}\right) v, x_{2}\right) Y\left(Y\left(g_{(2)}, x_{0}\right) h, x_{2}\right) w .
\end{aligned}
$$

Thus

$$
\begin{aligned}
& \left(x_{0}+x_{2}\right)^{l} Y_{W}\left(u \otimes g, x_{0}+x_{2}\right) Y_{W}\left(v \otimes h, x_{2}\right) w \\
= & \sum\left(x_{0}+x_{2}\right)^{l} Y\left(Y\left(u, x_{0}\right) Y\left(g_{(1)}, x_{0}\right) v, x_{2}\right) Y\left(Y\left(g_{(2)}, x_{0}\right) h, x_{2}\right) w .
\end{aligned}
$$

On the other hand, we have

$$
\begin{aligned}
& Y_{W}\left(Y_{\sharp}\left(u \otimes g, x_{0}\right)(v \otimes h), x_{2}\right) w \\
= & \sum Y_{W}\left(Y\left(u, x_{0}\right) Y\left(g_{(1)}, x_{0}\right) v \otimes Y\left(g_{(2)}, x_{0}\right) h, x_{2}\right) w \\
= & \sum Y\left(Y\left(u, x_{0}\right) Y\left(g_{(1)}, x_{0}\right) v, x_{2}\right) Y\left(Y\left(g_{(2)}, x_{0}\right) h, x_{2}\right) w .
\end{aligned}
$$

Therefore

$$
\begin{aligned}
& \left(x_{0}+x_{2}\right)^{l} Y_{W}\left(u \otimes g, x_{0}+x_{2}\right) Y_{W}\left(v \otimes h, x_{2}\right) w \\
= & \left(x_{0}+x_{2}\right)^{l} Y_{W}\left(Y_{\sharp}\left(u \otimes g, x_{0}\right)(v \otimes h), x_{2}\right) w .
\end{aligned}
$$

This proves that $W$ is a $V \sharp H$-module.

\section{$5 \quad$ Realizing vertex algebras $M(1)$ and $V_{L}$ through smash products}

In this section, we realize the vertex (operator) algebras associated with infinite-dimensional Heisenberg Lie algebras and the vertex algebras associated with nondegenerate even lattices through the smash product construction.

First we consider the vertex operator algebras associated with Heisenberg Lie algebras. Let $\mathbf{h}$ be a finite-dimensional vector space equipped with a nondegenerate symmetric bilinear form $\langle\cdot, \cdot\rangle$. Viewing $\mathbf{h}$ as an abelian Lie algebra we have the affine Lie algebra $\hat{\mathbf{h}}=\mathbf{h} \otimes \mathbb{C}\left[t, t^{-1}\right] \oplus \mathbb{C} c$, where

$$
\left[a \otimes t^{m}, b \otimes t^{n}\right]=m \delta_{m+n, 0}\langle a, b\rangle c
$$

for $a, b \in \mathbf{h}, m, n \in \mathbb{Z}$, and $c$ is a central element. For $h \in \mathbf{h}, n \in \mathbb{Z}$, following the tradition we also use $h(n)$ for $h \otimes t^{n}$. Set

$$
\hat{\mathbf{h}}^{-}=\mathbf{h} \otimes t^{-1} \mathbb{C}\left[t^{-1}\right],
$$


an abelian subalgebra of $\hat{\mathbf{h}}$. Let $L(-1)$ be the linear endomorphism of $\hat{\mathbf{h}}^{-}$defined by

$$
L(-1)(h(-n))=n h(-n-1) \quad \text { for } h \in \mathbf{h}, n \geq 1 .
$$

Of course, $L(-1)$ is a derivation of $\hat{\mathbf{h}}^{-}$as a Lie algebra. Set

$$
B_{\mathbf{h}}=U\left(\hat{\mathbf{h}}^{-}\right)=S\left(\hat{\mathbf{h}}^{-}\right),
$$

which is a vertex bialgebra by Example 4.3.

Next we are going to define a $B_{\mathbf{h}}$-module vertex algebra structure on $B_{\mathbf{h}}$. Notice that the adjoint module structure of $B_{\mathbf{h}}$ does not make $B_{\mathbf{h}}$ a $B_{\mathbf{h}}$-module vertex algebra.

For $\alpha \in \mathbf{h}$, set $\alpha(0)=0$ on $B_{\mathbf{h}}$. For $n \geq 1$, define $\alpha(n)$ to be the derivation of $B_{\mathbf{h}}$ with

$$
\alpha(n)(\beta(-m))=n\langle\alpha, \beta\rangle \delta_{n, m} \quad \text { for } \beta \in \mathbf{h}, m \geq 1 .
$$

Set

$$
\alpha(x)^{-}=\sum_{n \geq 0} \alpha(n) x^{-n-1} \in\left(\operatorname{Der} B_{\mathbf{h}}\right)\left[\left[x^{-1}\right]\right] .
$$

Lemma 5.1. For $\alpha \in \mathbf{h}$, we have $\alpha(x)^{-} \in \operatorname{PDer}^{-}\left(B_{\mathbf{h}}\right)$. There exists a $B_{\mathbf{h}}$-module structure $Y_{M}$ on $B_{\mathbf{h}}$, uniquely determined by

$$
Y_{M}(\alpha(-1), x)=\alpha(x)^{-} \quad \text { for } \alpha \in \mathbf{h} .
$$

Furthermore, $B_{\mathbf{h}}$ equipped with this $B_{\mathbf{h}}$-module structure $Y_{M}$ is a $B_{\mathbf{h}}$-module vertex algebra.

Proof. For $\alpha \in \mathbf{h}, u \in B_{\mathbf{h}}$, since $\alpha(n) u=0$ for $n$ sufficiently large, we have

$$
\alpha(x)^{-} u \in B_{\mathbf{h}}\left[x^{-1}\right] \subset B_{\mathbf{h}} \otimes \mathbb{C}((x)) .
$$

For $\alpha \in \mathbf{h}, n \geq 1$, with $L(-1)$ and $\alpha(n)$ being derivations of $B_{\mathbf{h}}$, it is straightforward to show that

$$
[L(-1), \alpha(n)]=-n \alpha(n-1) .
$$

That is, $\left[L(-1), \alpha(x)^{-}\right]=\frac{d}{d x} \alpha(x)^{-}$. By Proposition 2.11 we have $\alpha(x)^{-} \in \operatorname{PDer}^{-}\left(B_{\mathbf{h}}\right)$. For $\alpha, \beta \in L, h \in \mathbf{h}, m, n \geq 0$, it is also straightforward to show that

$$
\alpha(n) \beta(m)=\beta(m) \alpha(n) .
$$

Then $\alpha(x)^{-}$for $\alpha \in \mathbf{h}$ generate a commutative differential algebra in $B\left(B_{\mathbf{h}}\right)$. As $B_{\mathbf{h}}=$ $S\left(\hat{\mathbf{h}}^{-}\right)$is free, there exists a unique differential algebra homomorphism from $B_{\mathbf{h}}$ to $B\left(B_{\mathbf{h}}\right)$, sending $\alpha(-1)$ to $\alpha(x)^{-}$for $\alpha \in \mathbf{h}$. It follows from Lemma 4.6 that this homomorphism makes $B_{\mathbf{h}}$ a $B_{\mathbf{h}}$-module vertex algebra with $Y_{M}(\alpha(-1), x)=\alpha(x)^{-}$for $\alpha \in \mathbf{h}$. Since $B_{\mathbf{h}}$ as a vertex algebra is generated by $\alpha(-1)$ for $\alpha \in \mathbf{h}$, the uniqueness is clear. 
Now we fix this $B_{\mathbf{h}}$-module vertex algebra structure. Then we have the smash product

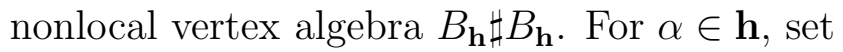

$$
\alpha(x)^{+}=\sum_{n \geq 1} \alpha(-n) x^{n-1} \in S\left(\hat{\mathbf{h}}^{-}\right)[[x]]=B_{\mathbf{h}}[[x]] .
$$

Recall that there exists a unique vertex algebra structure $Y_{M(1)}$ on $S\left(\hat{\mathbf{h}}^{-}\right)$with

$$
Y_{M(1)}(\alpha(-1), x)=\alpha(x)^{+}+\alpha(x)^{-}=\alpha(x) \quad \text { for } \alpha \in \mathbf{h} .
$$

This vertex algebra is denoted by $M_{\hat{\mathbf{h}}}(1,0)$ or by $M(1)$ for short.

Proposition 5.2. The associative subalgebra $\Delta\left(B_{\mathbf{h}}\right)$ of $B_{\mathbf{h}} \otimes B_{\mathbf{h}}$ is a vertex subalgebra of $B_{\mathbf{h}} \sharp B_{\mathbf{h}}$. Furthermore, $\Delta$ viewed as a map from $M_{\hat{\mathbf{h}}}(1,0)$ to $\Delta\left(B_{\mathbf{h}}\right)$,

$$
\Delta: M_{\hat{\mathbf{h}}}(1,0)=S\left(\hat{\mathbf{h}}^{-}\right)=B_{\mathbf{h}} \rightarrow \Delta\left(B_{\mathbf{h}}\right) \subset B_{\mathbf{h}} \otimes B_{\mathbf{h}}=B_{\mathbf{h}} \sharp B_{\mathbf{h}},
$$

is a vertex algebra isomorphism.

Proof. For $\alpha \in \mathbf{h}$, we have

$$
Y_{B_{\mathbf{h}}}(\alpha(-1), x)=e^{x L(-1)} \alpha(-1)=\sum_{n \geq 0} \alpha(-n-1) x^{n}=\alpha(x)^{+} .
$$

Then

$$
\begin{aligned}
Y^{\sharp}(\Delta(\alpha(-1)), x) & =Y^{\sharp}(\alpha(-1) \otimes 1+1 \otimes \alpha(-1), x) \\
& =Y_{B_{\mathbf{h}}}(\alpha(-1), x) \otimes 1+\alpha(x)^{-} \otimes 1+1 \otimes Y_{B_{\mathbf{h}}}(\alpha(-1), x) \\
& =\alpha(x)^{+} \otimes 1+\alpha(x)^{-} \otimes 1+1 \otimes \alpha(x)^{+} .
\end{aligned}
$$

Writing $Y^{\sharp}(u, x)=\sum_{n \in \mathbb{Z}} u_{n}^{\sharp}$ for $u \in B_{\mathbf{h}} \sharp B_{\mathbf{h}}$, we have

$$
\begin{array}{ll}
\Delta(\alpha(-1))_{-n}^{\sharp}=\alpha(-n) \otimes 1+1 \otimes \alpha(-n)=\Delta(\alpha(-n)) & \text { for } n \geq 1, \\
\Delta(\alpha(-1))_{m}^{\sharp}=\alpha(m) \otimes 1 & \text { for } m \geq 0 .
\end{array}
$$

Furthermore, for $\alpha, \beta \in \mathbf{h}, m, n \in \mathbb{Z}$, we have

$$
\left[\Delta(\alpha(-1))_{m}^{\sharp}, \Delta(\beta(-1))_{n}^{\sharp}\right]=m\langle\alpha, \beta\rangle \delta_{m+n, 0} .
$$

Then $B_{\mathbf{h}} \sharp B_{\mathbf{h}}$ is an $\hat{\mathbf{h}}$-module of level 1 with $\alpha(m)$ acting as $\Delta(\alpha(-1))_{m}^{\sharp}$ for $\alpha \in \mathbf{h}, m \in \mathbb{Z}$. Since $B_{\mathbf{h}}$ as an algebra is generated by $\alpha(-n)$ for $\alpha \in \mathbf{h}, n \geq 1$ and since $\Delta$ is an algebra homomorphism, it follows the P-B-W theorem and from (5.10) that $\Delta\left(B_{\mathbf{h}}\right)$ is exactly the $\hat{\mathbf{h}}$-submodule of $B_{\mathbf{h}} \sharp B_{\mathbf{h}}$ generated by $\mathbf{1} \otimes \mathbf{1}$. It then follows that $\Delta\left(B_{\mathbf{h}}\right)$ is exactly the nonlocal vertex subalgebra of $B_{\mathbf{h}} \sharp B_{\mathbf{h}}$ generated by $\Delta(\alpha(-1))$ for $\alpha \in \mathbf{h}$. In view of the P-B-W theorem, $\Delta$ is injective, so that $\Delta$, viewed as a map from $M_{\hat{\mathbf{h}}}(1,0)$ to $\Delta\left(B_{\mathbf{h}}\right)$, is an isomorphism of $\hat{\mathbf{h}}^{-}$-modules. Then from (5.12), $\Delta$, viewed as a map from $M_{\hat{\mathbf{h}}}(1,0)$ to $\Delta\left(B_{\mathbf{h}}\right)$, is an isomorphism of $\hat{\mathbf{h}}$-modules. Consequently, $\Delta$ is an isomorphism of vertex algebras. 
We next consider the vertex algebras associated with nondegenerate even lattices. Let $L$ be a nondegenerate even lattice; a free abelian group of finite rank equipped with a nondegenerate $\mathbb{Z}$-valued symmetric $\mathbb{Z}$-bilinear form $\langle\cdot, \cdot\rangle$ such that $\langle\alpha, \alpha\rangle \in 2 \mathbb{Z}$ for $\alpha \in L$. Set $\mathbf{h}=\mathbb{C} \otimes_{\mathbb{Z}} L$ and then linearly extend the form on $L$ to $\mathbf{h}$. As in the previous example we have an affine Lie algebra $\hat{\mathbf{h}}$ and a differential bialgebra $B_{\mathbf{h}}$. Let $\epsilon: L \times L \rightarrow \mathbb{C}^{\times}$be a map such that

$$
\begin{aligned}
& \epsilon(\alpha, 0)=\epsilon(0, \alpha)=1 \\
& \epsilon(\alpha, \beta+\gamma) \epsilon(\beta, \gamma)=\epsilon(\alpha+\beta, \gamma) \varepsilon(\alpha, \beta)
\end{aligned}
$$

for $\alpha, \beta, \gamma \in L$. Such a map is called a normalized $\mathbb{C}^{\times}$-valued 2-cocycle of $L$ (as an abelian group). Denote by $\mathbb{C}_{\epsilon}[L]$ the $\epsilon$-twisted group algebra of $L$ which has a $\mathbb{C}$-basis indexed by $L$, say $\left\{e_{\alpha} \mid \alpha \in L\right\}$, with multiplication

$$
e_{\alpha} e_{\beta}=\epsilon(\alpha, \beta) e_{\alpha+\beta} \quad \text { for } \alpha, \beta \in L \text {. }
$$

Set

$$
B_{L, \epsilon}=\mathbb{C}_{\epsilon}[L] \otimes B_{\mathbf{h}}
$$

an associative algebra. Extend the derivation $L(-1)$ of $B_{\mathbf{h}}$ to a linear endomorphism of $B_{L, \epsilon}$ by

$$
L(-1)\left(e_{\alpha} \otimes u\right)=e_{\alpha} \otimes \alpha(-1) u+e_{\alpha} \otimes L(-1) u
$$

for $\alpha \in L, u \in S\left(\hat{\mathbf{h}}^{-}\right)$. It is straightforward to check that $L(-1)$ is a derivation of $B_{L, \epsilon}$. This makes $B_{L, \epsilon}$ a differential algebra and hence a nonlocal vertex algebra.

Recall that we have operators $h(n)$ on $B_{\mathbf{h}}$ for $h \in \mathbf{h}, n \in \mathbb{Z}$. We extend the actions of $h(n)$ on $B_{\mathbf{h}}$ to $B_{L, \epsilon}$ by

$$
\begin{aligned}
& h(n)\left(e_{\alpha} \otimes u\right)=e_{\alpha} \otimes h(n) u \quad \text { for } n \neq 0, \\
& h(0)\left(e_{\alpha} \otimes u\right)=\langle h, \alpha\rangle e_{\alpha} \otimes u
\end{aligned}
$$

for $\alpha \in L, u \in B_{\mathbf{h}}$.

For $\alpha \in \mathbf{h}$, following [FLM] set

$$
E^{ \pm}(\alpha, x)=\exp \left(\sum_{n \in \pm \mathbb{Z}_{+}} \frac{\alpha(n)}{n} x^{-n}\right) .
$$

We have

$$
\begin{aligned}
& {\left[L(-1), E^{ \pm}(\alpha, x)\right]=-\left(\sum_{n \in \pm \mathbb{Z}_{+}} \alpha(n-1) x^{-n}\right) E^{ \pm}(\alpha, x),} \\
& \frac{d}{d x} E^{ \pm}(\alpha, x)=-\left(\sum_{n \in \pm \mathbb{Z}_{+}} \alpha(n) x^{-n-1}\right) E^{ \pm}(\alpha, x) .
\end{aligned}
$$


Lemma 5.3. For $\alpha \in L$, we have

$$
Y_{B_{L, \epsilon}}\left(e_{\alpha}, x\right)=E^{-}(-\alpha, x) e_{\alpha}
$$

where $Y_{B_{L, \epsilon}}$ denotes the vertex operator map of the nonlocal vertex algebra $B_{L, \epsilon}$.

Proof. For $\alpha \in L$, as $Y_{B_{L, \epsilon}}\left(e_{\alpha}, x\right)=e^{x L(-1)} e_{\alpha}$, we must prove $e^{-x L(-1)} E^{-}(-\alpha, x) e_{\alpha}=e_{\alpha}$. Clearly, it is true for $x=0$. Using the properties (5.18) and (5.19) we have

$$
\begin{aligned}
& \frac{d}{d x}\left(e^{-x L(-1)} E^{-}(-\alpha, x) e_{\alpha}\right) \\
= & -e^{-x L(-1)} L(-1) E^{-}(-\alpha, x) e_{\alpha}+e^{-x L(-1)}\left(\sum_{n \in-\mathbb{Z}_{+}} \alpha(n) x^{-n-1}\right) E^{-}(-\alpha, x) e_{\alpha} \\
= & -e^{-x L(-1)} E^{-}(-\alpha, x) L(-1) e_{\alpha}+e^{-x L(-1)} E^{-}(-\alpha, x) \alpha(-1) e_{\alpha} \\
= & 0,
\end{aligned}
$$

as $L(-1) e_{\alpha}=\alpha(-1) e_{\alpha}$. Thus $e^{-x L(-1)} E^{-}(-\alpha, x) e_{\alpha}=e_{\alpha}$.

Set

$$
B_{L}=\mathbb{C}[L] \otimes B_{\mathbf{h}}=\mathbb{C}[L] \otimes S\left(\hat{\mathbf{h}}^{-}\right),
$$

a Hopf algebra. Note that $B_{L}=B_{L, \epsilon}$ with $\epsilon$ being trivial. It is straightforward to check that the comultiplication $\Delta$ and the counit $\varepsilon$ are homomorphisms of differential algebras. Then $B_{L}$ is naturally a differential bialgebra. (Note that for a general $\epsilon, B_{L, \epsilon}$ is not a bialgebra with $\Delta$ defined in the obvious way.)

Next, we define a $B_{L}$-module nonlocal vertex algebra structure on $B_{L, \epsilon}$. For this purpose we need the following universal property of $B_{L}$ :

Lemma 5.4. Let $L$ be a nondegenerate even lattice. Let $A$ be any commutative associative algebra with a derivation $\partial$ and let $f: \mathbb{C}[L] \rightarrow A$ be any homomorphism of algebras. Then $f$ can be extended uniquely to a homomorphism of differential algebras from $B_{L}$ to $A$.

Proof. For $\alpha, \beta \in L$, we have

$$
\begin{aligned}
f\left(e^{-\alpha-\beta}\right) \partial f\left(e^{\alpha+\beta}\right) & =f\left(e^{-\alpha-\beta}\right) \partial\left(f\left(e^{\alpha}\right) f\left(e^{\beta}\right)\right) \\
& =f\left(e^{-\alpha-\beta}\right)\left(f\left(e^{\beta}\right) \partial f\left(e^{\alpha}\right)+f\left(e^{\alpha}\right) \partial f\left(e^{\beta}\right)\right) \\
& =f\left(e^{-\alpha}\right) \partial f\left(e^{\alpha}\right)+f\left(e^{-\beta}\right) \partial f\left(e^{\beta}\right) .
\end{aligned}
$$

In view of this, we have a linear map from $\mathbf{h}$ to $A$, sending $\alpha \in L$ to $f\left(e^{-\alpha}\right) \partial f\left(e^{\alpha}\right)$. Furthermore, we have an algebra homomorphism $g$ from $S\left(\hat{\mathbf{h}}^{-}\right)$to $A$ such that

$$
g(\alpha(-1-n))=\frac{1}{n !} \partial^{n}\left(f\left(e^{-\alpha}\right) \partial f\left(e^{\alpha}\right)\right) \quad \text { for } \alpha \in L, n \in \mathbb{N} .
$$

Then $f \otimes g$ is an algebra homomorphism from $B_{L}$ to $A$, extending $f$. Now we show that $(f \otimes g) L(-1)=\partial(f \otimes g)$, that is, $f \otimes g$ is a homomorphism of differential algebras. For $\alpha \in$ 
$L, n \in \mathbb{N}$, since $\alpha(-n-1)=\frac{1}{n !} L(-1)^{n} \alpha(-1)$, we have $g\left(L(-1)^{n} \alpha(-1)\right)=\partial^{n} g(\alpha(-1))$. Assume that $a, b \in B_{\mathbf{h}}$ such that $g L(-1)(a)=\partial g(a)$ and $g L(-1)(b)=\partial g(b)$. Then

$$
g L(-1)(a b)=g(a L(-1) b)+g(b L(-1) a)=g(a) \partial g(b)+g(b) \partial(a)=\partial(g(a) g(b))=\partial g(a b) .
$$

It follows from induction that $g L(-1)=\partial g$. Furthermore, for $\alpha \in L$, we have

$$
(f \otimes g) L(-1) e^{\alpha}=(f \otimes g)\left(e^{\alpha} \otimes \alpha(-1)\right)=f\left(e^{\alpha}\right) f\left(e^{-\alpha}\right) \partial f\left(e^{\alpha}\right)=\partial f\left(e^{\alpha}\right)=\partial(f \otimes g) e^{\alpha} .
$$

Then it follows that $(f \otimes g) L(-1)=\partial(f \otimes g)$. As $\mathbb{C}[L]$ generates $B_{\mathbf{h}}$ as a differential algebra, the uniqueness is clear.

Proposition 5.5. We have

$$
E^{+}(-\alpha, x) x^{\alpha(0)} \in \operatorname{PEnd}^{-}\left(B_{L, \epsilon}\right) \quad \text { for } \alpha \in L .
$$

Furthermore, there exists a unique $B_{L}$-module structure $Y_{M}$ on $B_{L, \epsilon}$ such that

$$
Y_{M}\left(e^{\alpha}, x\right)=E^{+}(-\alpha, x) x^{\alpha(0)} \quad \text { for } \alpha \in L
$$

and $B_{L, \epsilon}$ equipped with this $B_{L}$-module structure $Y_{M}$ is a $B_{L}$-module nonlocal vertex algebra.

Proof. Set $\Phi_{\alpha}(x)=E^{+}(-\alpha, x) x^{\alpha(0)}$ for $\alpha \in L$. It is straightforward to check that for $\alpha \in L, n \geq 0, \alpha(n) \in \operatorname{Der}\left(B_{L, \epsilon}\right)$ and $[L(-1), \alpha(n)]=-n \alpha(n-1)$. Then

$$
\left[L(-1), \log \Phi_{\alpha}(x)\right]=\frac{d}{d x} \log \Phi_{\alpha}(x) .
$$

In view of Proposition 2.11, we have

$$
\log \Phi_{\alpha}(x) \in \operatorname{Der}\left(B_{L, \epsilon}, B_{L, \epsilon} \otimes(\mathbb{C}((x))[\log x],-d / d x)\right) .
$$

Consequently, $\Phi_{\alpha}(x) \in \operatorname{PEnd}^{-}\left(B_{L, \epsilon}\right)$. Clearly, we have $\Phi_{0}(x)=1$ and

$$
\Phi_{\alpha}(x) \Phi_{\beta}(x)=\Phi_{\alpha+\beta}(x) \quad \text { for } \alpha, \beta \in L .
$$

Denote by $A$ the subalgebra of $B\left(B_{L, \epsilon}\right)$ generated by $(d / d x)^{n} \Phi_{\alpha}(x)$ for $n \in \mathbb{N}, \alpha \in L$. It is clear that $A$ is a commutative differential subalgebra. By Lemma 5.4 there exists a homomorphism $\pi$ of differential algebras from $B_{L}$ to $A$ such that $\pi\left(e^{\alpha}\right)=\Phi_{\alpha}(x)$ for $\alpha \in L$. As $B_{L, \varepsilon}$ is a module for $B\left(B_{L, \varepsilon}\right)$ as a nonlocal vertex algebra (by Proposition [3.4), the homomorphism $\pi$ gives rise to a $B_{L}$-module structure $Y_{M}$ on $B_{L, \varepsilon}$ such that $Y_{M}\left(e^{\alpha}, x\right)=\Phi_{\alpha}(x)$ for $\alpha \in L$. It follows from Lemma 4.6 that $B_{L, \epsilon}$ equipped with the $B_{L}$-module structure $Y_{M}$ is a $B_{L}$-module nonlocal vertex algebra. As $\mathbb{C}[L]$ generates $B_{L}$ as a vertex algebra, the uniqueness is clear.

Now we fix the $B_{L}$-module nonlocal vertex algebra structure on $B_{L, \epsilon}$. We have the smash product nonlocal vertex algebra $B_{L, \epsilon \sharp} B_{L}$. 
Remark 5.6. Recall from [FLM] that for the vertex algebra $V_{L}$ associated with $L$, we have $V_{L}=B_{L, \epsilon}=\mathbb{C}_{\epsilon}[L] \otimes S\left(\hat{\mathbf{h}}^{-}\right)$as a vector space, where $\epsilon$ is a normalized 2-cocycle of $L$ satisfying the condition

$$
\epsilon(\alpha, \beta) \epsilon(\beta, \alpha)^{-1}=(-1)^{\langle\alpha, \beta\rangle} \quad \text { for } \alpha, \beta \in L .
$$

Denote the vertex operator map of $V_{L}$ by $Y_{V_{L}}$. For $\alpha \in L$, we have

$$
Y_{V_{L}}\left(e_{\alpha}, x\right)=E^{-}(-\alpha, x) E^{+}(-\alpha, x) e_{\alpha} \cdot x^{\alpha(0)}
$$

Proposition 5.7. Let $L$ be a nondegenerate even lattice and let $\epsilon$ be the normalized 2cocycle of $L$, which was used in the construction of the vertex algebra $V_{L}$. Set

$$
U=\coprod_{\alpha \in L} \mathbb{C}\left(e_{\alpha} \otimes e^{\alpha}\right) \otimes \Delta\left(B_{\mathbf{h}}\right) \subset B_{L, \epsilon \sharp} B_{L} .
$$

Then $U$ is an ordinary vertex subalgebra of $B_{L, \epsilon} \sharp B_{L}$ and the linear map

$$
\pi: V_{L} \rightarrow U ; \quad e_{\alpha} \otimes u \mapsto\left(e_{\alpha} \otimes e^{\alpha}\right) \otimes \Delta(u)
$$

for $\alpha \in L, u \in S\left(\hat{\mathbf{h}}^{-}\right)$is an isomorphism of vertex algebras.

Proof. With $\Delta$ being an isomorphism from $B_{\mathbf{h}}$ to $B_{\mathbf{h}} \otimes B_{\mathbf{h}}$, it is clear that $\pi$ is a linear isomorphism. Then it remains to prove that $\pi$ is a homomorphism of vertex algebras. Let $\alpha, \beta \in L, u \in S\left(\hat{\mathbf{h}}^{-}\right)=B_{\mathbf{h}}$. From Remark 5.6 we have

$$
Y_{V_{L}}\left(e_{\alpha}, x\right)\left(e^{\beta} \otimes u\right)=x^{\langle\alpha, \beta\rangle} \epsilon(\alpha, \beta)\left(e_{\alpha+\beta} \otimes E^{-}(-\alpha, x) E^{+}(-\alpha, x) u\right) .
$$

Using (5.10) and (5.11) we get

$$
\begin{aligned}
& \pi\left(Y_{V_{L}}\left(e_{\alpha}, x\right)\left(e^{\beta} \otimes u\right)\right) \\
= & x^{\langle\alpha, \beta\rangle} \epsilon(\alpha, \beta)\left(e_{\alpha+\beta} \otimes e^{\alpha+\beta}\right) \Delta\left(E^{-}(-\alpha, x) E^{+}(-\alpha, x) u\right) \\
= & x^{\langle\alpha, \beta\rangle} \epsilon(\alpha, \beta)\left(e_{\alpha+\beta} \otimes e^{\alpha+\beta}\right)\left(E^{-}(-\alpha, x) E^{+}(-\alpha, x) \otimes E^{-}(-\alpha, x)\right) \Delta(u) .
\end{aligned}
$$

On the other hand, as $\Delta\left(e^{\alpha}\right)=e^{\alpha} \otimes e^{\alpha}$, using Lemma 5.3 we have

$$
\begin{aligned}
Y^{\sharp}\left(e_{\alpha} \otimes e^{\alpha}, x\right) & =Y_{B_{L, \epsilon}}\left(e_{\alpha}, x\right) \Phi_{\alpha}(x) \otimes Y_{B_{L}}\left(e^{\alpha}, x\right) \\
& =E^{-}(-\alpha, x) e_{\alpha} E^{+}(-\alpha, x) x^{\alpha(0)} \otimes E^{-}(-\alpha, x) e^{\alpha} .
\end{aligned}
$$

Then

$$
\begin{aligned}
& Y^{\sharp}\left(e_{\alpha} \otimes e^{\alpha}, x\right) \psi\left(e_{\beta} \otimes u\right) \\
= & Y^{\sharp}\left(e_{\alpha} \otimes e^{\alpha}, x\right)\left(e_{\beta} \otimes e^{\beta}\right) \Delta(u) \\
= & x^{\langle\alpha, \beta\rangle} \epsilon(\alpha, \beta)\left(e_{\alpha+\beta} \otimes e^{\alpha+\beta}\right)\left(E^{-}(-\alpha, x) E^{+}(-\alpha, x) \otimes E^{-}(-\alpha, x)\right) \Delta(u) .
\end{aligned}
$$

Consequently, we have

$$
\pi\left(Y_{V_{L}}\left(e_{\alpha}, x\right)\left(e^{\beta} \otimes u\right)\right)=Y^{\sharp}\left(e_{\alpha} \otimes e^{\alpha}, x\right) \pi\left(e_{\beta} \otimes u\right)
$$

for $\alpha, \beta \in L, u \in S\left(\hat{\mathbf{h}}^{-}\right)=B_{\mathbf{h}}$. As $\mathbb{C}_{\epsilon}[L]$ generates $V_{L}$ as a vertex algebra, $\pi$ is a homomorphism of nonlocal vertex algebras. Since $V_{L}$ is a vertex algebra, $\pi$ is an isomorphism of vertex algebras. 
Remark 5.8. Let $P$ be the dual lattice of $L$, i.e.,

$$
P=\{h \in \mathbf{h} \mid\langle\alpha, h\rangle \in \mathbb{Z} \quad \text { for all } \alpha \in L\} .
$$

Extend $\epsilon$ to a map from $L \times P \rightarrow \mathbb{C}^{\times}$such that

$$
\epsilon(\alpha, \beta) \epsilon(\alpha+\beta, \gamma)=\epsilon(\alpha, \beta+\gamma) \epsilon(\beta, \gamma)
$$

for $\alpha, \beta \in L, \gamma \in P$. Define a $\mathbb{C}_{\epsilon}[L]$-module structure on $\mathbb{C}[P]$ by

$$
e_{\alpha} \cdot e^{\gamma}=\epsilon(\alpha, \gamma) e^{\alpha+\gamma} \quad \text { for } \alpha \in L, \gamma \in P \text {. }
$$

Set

$$
V_{P}=\mathbb{C}[P] \otimes B_{\mathbf{h}}=\mathbb{C}[P] \otimes S\left(\hat{\mathbf{h}}^{-}\right) .
$$

The space $V_{P}$ is naturally a $B_{L, \epsilon}$-module. On the other hand, the same argument in Proposition 5.5 shows that there exists a (unique) $B_{L}$-module structure $Y_{M}$ on $V_{P}$ with $Y_{M}\left(e^{\alpha}, x\right)=E^{+}(-\alpha, x) x^{\alpha(0)}$ for $\alpha \in L$. Using the commutation relation between $E^{+}\left(\alpha, x_{1}\right)$ and $E^{-}\left(\beta, x_{2}\right)$ (see [FLM]) one sees that Proposition 4.11] applies to our situation, so $V_{P}$ is a $B_{L, \epsilon} \sharp B_{L}$-module. As $V_{L}$ is a vertex subalgebra of $B_{L, \epsilon} \sharp B_{L}, V_{P}$ is a $V_{L}$-module. This gives a new proof for the existence of a $V_{L}$-structure on $V_{P}$ (cf. [FLM], [LL).

\section{References}

[BK] B. Bakalov and V. Kac, Field algebras, Internat. Math. Res. Notices 3 (2003), 123-159.

[B1] R. E. Borcherds, Vertex algebras, Kac-Moody algebras, and the Monster, Proc. Natl. Acad. Sci. USA 83 (1986), 3068-3071.

[B2] R. E. Borcherds, Vertex algebras, in "Topological Field Theory, Primitive Forms and Related Topics" (Kyoto, 1996), edited by M. Kashiwara, A. Matsuo, K. Saito and I. Satake, Progress in Math. 160, Birkhäuser, Boston, 1998, 35-77.

[B3] R. E. Borcherds, Quantum vertex algebras, preprint, arXiv:math.QA/9903038.

[DLM] C. Dong, H.-S. Li and G. Mason, Vertex Lie algebras, Poisson algebras and vertex operator algebras, Contemporary Math. 297, Amer. Math. Soc., 2002, 69-96.

[EK] P. Etingof and D. Kazhdan, Quantization of Lie bialgebras, V, Selecta Mathematica, New Series, 6 (2000), 105-130.

[FB] E. Frenkel and D. Ben-Zvi, Vertex Algebras and Algebraic Curves, Mathematical Surveys and Monographs, Vol. 88, Amer. Math. Soc., Providence, 2001. 
[FHL] I. Frenkel, Y.-Z. Huang and J. Lepowsky, On axiomatic approaches to vertex operator algebras and modules, Memoirs Amer. Math. Soc. 104, 1993.

[FLM] I. Frenkel, J. Lepowsky and A. Meurman, Vertex Operator Algebras and the Monster, Pure and Appl. Math., Vol. 134, Academic Press, Boston, 1988.

[H1] K. Hubbard, The notion of vertex operator coalgebra and a geometric interpretation, math.QA/0405461.

[H2] K. Hubbard, Constructions of vertex operator coalgebras via vertex operator algebras, math.QA/0406035.

[K] V. Kac, Vertex Algebras for Beginners, University Lecture Series 10, Amer. Math. Soc., 1997.

[LL] J. Lepowsky and H.-S. Li, Introduction to Vertex Operator Algebras and Their Representations, Progress in Math. 227, Birkhäuser, Boston, 2004.

[Li1] H.-S. Li, Local systems of twisted vertex operators, vertex superalgebras and twisted modules, Contemporary Math. 193 (1996), 203-236.

[Li2] H.-S. Li, The physics superselection principle in vertex operator algebra theory, J. Algebra 196 (1997), 436-457.

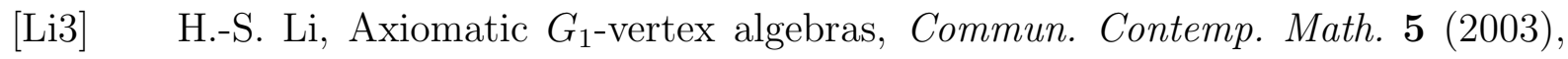
281-327.

[Li4] H.-S. Li, Simple vertex operator algebras are nondegenerate, J. Algebra 267 (2003), 199-211.

[Li5] H.-S. Li, Pseudoderivations, pseudoautomorphisms and simple current modules for vertex operator algebras, in Proceedings of the International Conference on "Infinite Dimensional Aspects of Representation Theory and Applications," University of Virginia, Charlottesville, May 18-22, 2004.

[Li6] H.-S. Li, Constructing quantum vertex algebras, International Journal of Mathematics, to appear; math.QA/0505294.

[M] S. Montgomery, Hopf Algebras and Their Actions on Rings, CBMS Regional Conference Series in Mathematics, Number 82, AMS, 1993.

[P] M. Primc, Vertex algebras generated by Lie algebras, J. Pure Appl. Alg. 135 (1999), 253-293. 